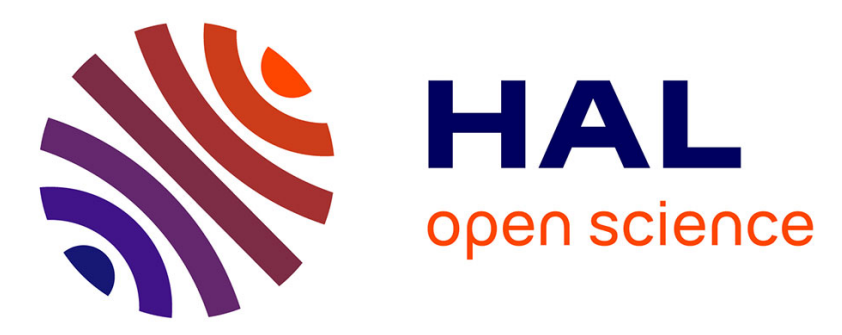

\title{
Modelling for isothermal cavitation with a four-equation model
}

Eric Goncalvès da Silva, Boris Charrière

\section{To cite this version:}

Eric Goncalvès da Silva, Boris Charrière. Modelling for isothermal cavitation with a four-equation model. International Journal of Multiphase Flow, 2014, 59, pp.54-72. 10.1016/j.ijmultiphaseflow.2013.10.015 . hal-00912579

HAL Id: hal-00912579

https://hal.science/hal-00912579

Submitted on 2 Dec 2013

HAL is a multi-disciplinary open access archive for the deposit and dissemination of scientific research documents, whether they are published or not. The documents may come from teaching and research institutions in France or abroad, or from public or private research centers.
L'archive ouverte pluridisciplinaire HAL, est destinée au dépôt et à la diffusion de documents scientifiques de niveau recherche, publiés ou non, émanant des établissements d'enseignement et de recherche français ou étrangers, des laboratoires publics ou privés. 


\title{
Modeling for isothermal cavitation with a four-equation model
}

\author{
Eric Goncalvès*, Boris Charrière
}

LEGI-University of Grenoble, 1025 rue de la Piscine, 38400 St Martin d'Heres, France

\begin{abstract}
In a recent study, an original formulation for the mass transfer between phases has been proposed to study one-dimensional inviscid cavitating tube problems. This mass transfer term appears explicitly as a source term of a void ratio transportequation model in the framework of the homogenous mixture approach. Based on this generic form, a two-dimensional preconditioned Navier-Stokes one-fluid solver is developed to perform realistic cavitating flows. Numerical results are given for various inviscid cases (underwater explosion, bubble collapse) and unsteady sheet cavitation developing along Venturi geometries at high Reynolds number. Comparisons with experimental data (concerning void ratio and velocity profiles, pressure fluctuations) and with a 3-equation model are presented.
\end{abstract}

Keywords: Cavitation, Mass transfer, Homogeneous model, RANS simulation

\section{Introduction}

Cavitation is a significant engineering phenomenon that occurs in fluid machinery, fuel injectors, marine propellers, nozzles, underwater bodies, etc. In most cases, cavitation is an undesirable phenomenon, significantly degrading performance, resulting in reduced flow rates, lower pressure increases in pumps, load asymmetry,

\footnotetext{
${ }^{*}$ Corresponding author.

Email address: Eric.Goncalves@legi.grenoble-inp.fr (Eric Goncalvès )
} 
vibrations, noise and erosion. In most industrial applications, cavitating flows are turbulent and the dynamics of the interface formed involves complex interactions between the vapour and liquid phases. These interactions are not well understood in the closure region of cavities, where a distinct interface may not exist and where the flow is unsteady.

Several physical and numerical models have been developed to investigate cavitating flows within the framework of averaged two-phase model. For the averaged model, there are different approaches according to the assumptions made on the local thermodynamic equilibrium and the slip condition between phases. A hierarchy of models exists, with the numbers of equations ranging from seven to three only. The full non-equilibrium two-fluid models with relaxation procedures have been tested on inviscid high-speed applications (see for example (Petitpas et al., 2009; Zein et al., 2010)), whereas one-fluid models have been massively used for industrial cavitating flows.

By assuming the velocity, pressure and thermal equilibrium between phases, various formulations of four-equation model have been expressed. A very popular formulation has been developed to simulate turbulent cavitating flows (Merkle et al., 1998; Kunz et al., 2000; Senocak and Shyy, 2002; Singhal et al., 2002; Venkateswaran et al., 2002; Vortmann et al., 2003; Wu et al., 2005; Wang and Ostoja-Starzewski, 2007; Morgut et al., 2011; Ji et al., 2012). It is composed by three conservation laws for mixture quantities (mass, momentum, energy) plus a mass equation for the vapour or liquid density including a cavitation source term. The main difficulty is related to the formulation of the source term and the tunable parameters involved for the vaporization and condensation processes. Moreover, this family of models are not thermodynamically well-posed and does not respect thermodynamic constraints 
(Goncalves and Patella, 2011). Another approach of source term term was proposed in (Helluy and Seguin, 2006), based on a constrained convex optimization problem on the mixture entropy.

With the assumption of complete thermodynamic equilibrium between phases (local temperature, pressure and free Gibbs enthalpy equality between phases), we obtain the 3-equation models or homogeneous equilibrium models (HEM). Vaporization or condensation processes are assumed to be instantaneous. An equation of state (EOS) is necessary to close the system. Different closure relations (tabulated EOS or combination of pure phase EOSs) that link the pressure to the thermodynamic variables have been proposed (Delannoy and Kueny, 1990; Saurel et al., 1999; Schmidt et al., 1999; Ventikos and Tzabiras, 2000; Liu et al., 2004; Schmidt et al., 2006; Sinibaldi et al., 2006; Ihm and Kim, 2008; Goncalves and Patella, 2009).

In addition, the turbulence modelling plays a determinant role in the capture of unsteady behaviours. Cavitation sheets that appear on solid bodies are characterized by a closure region which always fluctuates with the existence of a re-entrant jet. This one is mainly composed of liquid which flows upstream along the solid surface. Moreover, compressibility effects on turbulence are involved. These effects and interactions with two-phase structures are not yet well known and understood. For usual applications, three-dimensional time-dependent computations obtained with large eddy simulations (LES) or direct simulations (DNS) are not yet tractable. The Reynolds decomposition is often used with an averaged statistical processing resulting in the RANS equations for the mean flow quantities. The limitation of the turbulent viscosity evaluated with transport-equation turbulence models (through the Boussinesq assumption) is a key point to capture realistic cavitation sheets. Different methods have been investigated to limit or to correct standard turbulence models. 
One of the most popular limiter was proposed by Reboud to reduce the turbulent viscosity (Reboud et al., 1998), and has successfully been used by different authors (Coutier-Delgosha et al., 2002; Chen et al., 2006; Zhou and Wang, 2008; Srinivasan et al., 2009; Goncalves, 2011).

In a recent study, an original source term including the mass transfer between phases was proposed using a void ratio transport-equation model. A particular emphasis was placed on the thermodynamic coherence. The mass transfer was closed assuming its proportionality to the divergence of the homogeneous velocity field. First validations on one-dimensional rarefaction tube problems showed the good behaviour of the model and the low sensitivity to the involved constant (Goncalves, 2012, 2013). In the present paper, the cavitation model is improved and implemented in a compressible two-dimensional RANS/Euler solver. This new formulation is firstly tested on inviscid test cases (solving the compressible one-fluid Euler equations) such as underwater explosion and bubble collapse. Secondly, two turbulent sheets cavitation appearing on Venturi geometries are simulated and compared with the available experimental data (time-averaged void ratio and velocity profiles, pressure fluctuations, oscillation frequency). The influence of the constant is investigated, especially the effect on the sheet cavitation dynamic. Moreover, a comparison with a 3-equation model is proposed.

This paper is organized as follows. We first review the theoretical formulation including physical models, the mass transfer formulation and elements of the numerical methods. The preliminary studies carried out in inviscid test cases are presented. This is followed by sets of results on two turbulent Venturi flows and discussions. 


\section{Governing equations and models}

The numerical simulations are carried out using an in-house CFD code solving the one-fluid compressible RANS or Euler system.

\subsection{The homogeneous approach}

The homogeneous mixture approach is used to model two-phase flows. The phases are assumed to be sufficiently well mixed and the disperse particle size are sufficiently small thereby eliminating any significant relative motion. The phases are strongly coupled and moving at the same velocity. In addition, the phases are assumed to be in thermal and mechanical equilibrium: they share the same temperature $T$ and the same pressure $P$. The evolution of the two-phase flow can be described by the conservation laws that employ the representative flow properties as unknowns just as in a single-phase problem.

We introduce $\alpha_{k}$ the void fraction or the averaged fraction of presence of phase $k$. The density $\rho$, the center of mass velocity $u$ and the internal energy $e$ for the mixture are defined by (Ishii and Hibiki, 2006):

$$
\begin{aligned}
\rho & =\sum_{k} \alpha_{k} \rho_{k} \\
\rho u_{i} & =\sum_{k} \alpha_{k} \rho_{k} u_{i, k} \\
\rho e & =\sum_{k} \alpha_{k} \rho_{k} e_{k}
\end{aligned}
$$

\subsection{The mixture equation of state}

To close the compressible system, an equation of state (EOS) is necessary to link the pressure to the thermodynamic variables. Pure phases follow the stiffened gas EOS. The barotropic law (Delannoy and Kueny, 1990; Goncalves and Patella, 2009) 
is considered for the mixture. When the pressure is between $P_{v a p}(T)+\Delta P$ and $P_{v a p}(T)-\Delta P$, the following relationship applies:

$$
P(\rho)=P_{\text {vap }}+\left(\frac{\rho_{L}^{s a t}-\rho_{V}^{s a t}}{2}\right) c_{\text {baro }}^{2} \operatorname{Arcsin}\left(\frac{2 \rho-\rho_{L}^{s a t}-\rho_{V}^{s a t}}{\rho_{L}^{s a t}-\rho_{V}^{s a t}}\right)
$$

where $\Delta P$ represents the pressure range of the law and, for a void ratio value of 0.5 , the pressure is equal to the saturation pressure $P_{v a p}$ at the reference temperature. The cavitation phenomenon is assumed to be isothermal, thermal effects are neglected.

The associated speed of sound in the mixture is

$$
c^{2}=\left(\frac{\partial P}{\partial \rho}\right)_{s}=\left(\frac{\partial P}{\partial \rho}\right)_{T}=\frac{c_{\text {baro }}^{2}}{2 \sqrt{\alpha(1-\alpha)}}
$$

Properties of the model (such as convexity conditions of the EOS) and the influence of the parameter $c_{\text {baro }}$ have been studied in (Goncalves and Patella, 2009).

\subsection{A four-equation model with mass transfer}

The model consists in three conservation laws for mixture quantities (mass, momentum and total energy) and an additional equation for the void ratio. It is obtained from a reduction of a 5-equation model (Goncalves, 2013). The expression for the void ratio equation is:

$$
\frac{\partial \alpha}{\partial t}+\operatorname{div}(\alpha V)=(\underbrace{\left(\frac{\rho_{l} c_{l}^{2}-\rho_{v} c_{v}^{2}}{\frac{\rho_{l} c_{l}^{2}}{1-\alpha}+\frac{\rho_{v} c_{v}^{2}}{\alpha}}\right)}_{=K}+\alpha) \operatorname{div} V+\underbrace{\left(\frac{\frac{c_{v}^{2}}{\alpha}+\frac{c_{l}^{2}}{1-\alpha}}{\frac{\rho_{l} c_{l}^{2}}{1-\alpha}+\frac{\rho_{v} c_{v}^{2}}{\alpha}}\right)}_{=1 / \rho_{I} \text { the interfacial density }} \dot{m}
$$

where $\dot{m}$ is the mass transfer between phases and $V$ the velocity vector. The term $K$ involves the speed of sound of pure phases $c_{k}$ and it reflects the effects of changes 
in volume of each phase.

By assuming that the mass transfer is proportional to the divergence of the velocity, it is possible to build a family of models in which $\dot{m}$ is expressed as (the demonstration is presented in (Goncalves, 2013))

$$
\dot{m}=\frac{\rho_{l} \rho_{v}}{\rho_{l}-\rho_{v}}\left(1-\frac{c^{2}}{c_{\text {wallis }}^{2}}\right) \operatorname{div} V
$$

where $c_{\text {wallis }}$ is the propagation of acoustic waves without mass transfer (Wallis, 1967). This speed is expressed as the weighted harmonic mean of speeds of sound of each phase:

$$
\frac{1}{\rho c_{\text {wallis }}^{2}}=\frac{\alpha}{\rho_{v} c_{v}^{2}}+\frac{1-\alpha}{\rho_{l} c_{l}^{2}}
$$

When heat and mass transfer effects are involved in the flow, the sound speed $c$ decreases to the thermodynamic equilibrium one (Petitpas et al., 2009). This limit speed is evaluated with the assumption of local thermodynamic equilibrium: equalities of pressure, temperature and free enthalpy.

With this generic form for the mass transfer, we remark that all models in which the mixture speed of sound is the Wallis one cannot produce void ratio during the phase transition (that is the case of most of models proposed in the literature). The void ratio is only modified through the term $K \operatorname{div} V$.

A first model was built using the barotropic speed of sound and validated on inviscid rarefaction tube problems by comparisons with two-fluid solutions (Goncalves, 2013).

Yet, the simulation for turbulent cavitating cases put in evidence a problem with this model. Downstream the sheet cavities, the convected void ratio is not destroyed. The 
model is able to produce and to transport the void ratio but it does not destroy the void ratio in the flow where the pressure is greater than the vapour pressure $P_{\text {vap }}$. We propose to modify the formulation adding a destruction term

$$
\dot{m}=\frac{\rho_{l} \rho_{v}}{\rho_{l}-\rho_{v}}\left(1-\frac{c^{2}}{c_{\text {wallis }}^{2}}\right) \operatorname{div} V-C_{\text {des }} \frac{\rho_{v}}{\rho_{l}} \alpha \frac{\operatorname{Max}\left(0, P-P_{\text {vap }}\right)}{0.5 \rho_{\text {ref }} U_{\text {ref }}^{2}}
$$

where $C_{d e s}$ is a tunable parameter.

This new formulation does not modify the mixture speed of sound $c$ and the eigenvalues of the inviscid system. The system is hyperbolic with the characteristic waves speeds: $u-c, u, u, u, u+c$ where $c$ is the barotropic speed of sound.

\subsection{Reynolds-Averaged Navier-Stokes equations}

For turbulent computations, the compressible one-fluid RANS equations are used, coupled with a one- or two-equation turbulence model. For low Mach number applications, an inviscid preconditioner is introduced. These equations can be expressed as:

$$
\begin{gathered}
P_{c}^{-1} \frac{\partial w}{\partial t}+\operatorname{div}\left(F_{c}-F_{v}\right)=S \\
w=\left(\begin{array}{c}
\rho \\
\rho V \\
\rho E \\
\alpha \\
\rho k \\
\rho \Psi
\end{array}\right) \quad ; \quad F_{c}=\left(\begin{array}{c}
\rho V \\
\rho V \otimes V+p \overline{\bar{I}} \\
(\rho E+p) V \\
\alpha V \\
\rho k V \\
\rho \Psi V
\end{array}\right) \quad ; F_{v}=\left(\begin{array}{c}
10) \\
0 \\
\left(\overline{\overline{\tau^{v}}}+\overline{\overline{\tau^{t}}}\right) \cdot V-Q^{v}-Q^{t} \\
0 \\
\left(\mu+\mu_{t} / \sigma_{k}\right) \operatorname{grad} k \\
\left(\mu+\mu_{t} / \sigma_{\Psi}\right) \operatorname{grad} \Psi
\end{array}\right)
\end{gathered}
$$

where $w$ denotes the conservative variables and the void ratio, $F_{c}$ and $F_{v}$ the convective and viscous flux densities and $S$ the source terms, which concern the void ratio 
equation and the turbulent transport equations. $k$ is the mixture turbulent kinetic energy (TKE) and $\Psi$ is a mixture turbulent variable. In multiphase flow, the divergence of the fluctuating phase velocity is not zero (Decaix and Goncalves, 2013). Therefore, supplementary terms appear in the mixture TKE equation (pressuredilation term, dilatational dissipation rate), which are not taken into account in the present paper.

The exact expression of the eddy-viscosity $\mu_{t}$ and the source terms depends on the turbulence model as well as constants $\sigma_{k}$ and $\sigma_{\Psi}$.

The total stress tensor $\overline{\bar{\tau}}$ is evaluated using the Stokes hypothesis, Newton's law and the Boussinesq assumption. The total heat flux vector $Q$ is obtained from the Fourier law involving a turbulent thermal conductivity $\lambda_{t}$ with the constant Prandtl number hypothesis.

$$
\begin{aligned}
& \overline{\bar{\tau}}=\overline{\overline{\tau^{v}}}+\overline{\overline{\tau^{t}}}=\left(\mu+\mu_{t}\right)\left[\left(\operatorname{grad} V+(\operatorname{grad} V)^{t}\right)-\frac{2}{3}(\operatorname{div} V) \overline{\bar{I}}\right]+\frac{2}{3} \rho k \overline{\bar{I}} \\
& Q=Q^{v}+Q^{t}=-\left(\lambda+\lambda_{t}\right) \operatorname{grad} T \quad \text { with } \quad \lambda_{t}=\frac{\mu_{t} C_{p}}{P_{r t}}
\end{aligned}
$$

In pure phases, the viscosity is assumed to be constant. The mixture viscosity is defined as the arithmetic mean of the liquid and vapour viscosities (fluctuations of viscosity are neglected) (Ishii and Hibiki, 2006):

$$
\mu(\alpha)=\alpha \mu_{V}+(1-\alpha) \mu_{L}
$$

The mixture thermal conductivity $\lambda$ is also defined as the arithmetic mean of the liquid and vapour values:

$$
\lambda(\alpha)=\alpha \frac{\mu_{V} C_{p_{V}}}{P_{r_{V}}}+(1-\alpha) \frac{\mu_{L} C_{p_{L}}}{P_{r_{L}}}
$$

The turbulent Prandtl number $P_{r t}$ is set to 1 . 


\subsection{The turbulence model}

The present study is based on the one-equation Spalart-Allmaras (SA) turbulence model (Spalart and Allmaras, 1992) in witch the Reboud correction is added.

\subsection{Wall functions}

For the modelling of flow close to the wall, a two-layer wall law approach is used:

$$
\begin{array}{rlrl}
u^{+} & =y^{+} & \text {if } & y^{+}<11.13 \\
u^{+} & =\frac{1}{\kappa} \ln y^{+}+5.25 & \text { if } & y^{+}>11.13 \\
u^{+}=\frac{u}{U_{\tau}} & ; & y^{+}=\frac{y U_{\tau}}{\nu_{w}} \quad ; \quad U_{\tau}^{2}=\frac{\tau_{w}}{\rho_{w}}
\end{array}
$$

where $\kappa=0.41$ is the von Karman constant and the subscript 'w' is used for a wall value.

We assume that wall functions are similar in a two-phase flow and in a single-phase flow. For unsteady flows, the existence of a wall law is assumed to be valid at each instant. These assumptions have been studied in (Goncalves and Decaix, 2012) and comparisons were proposed with a thin boundary layer approach.

With regard to the turbulent quantities, the production of $k$ is computed according to the formulation proposed by Viegas and Rubesin (Viegas and Rubesin, 1983). The value of $\ell$ in the first cell is obtained using a classical mixing length: $l=\kappa y$.

\section{Numerical methods}

The numerical simulations are carried out using an implicit solver for multidomain structured meshes. This solver is based on a cell-centered finite-volume discretization. 


\subsection{Spatial discretization}

For the mean flow, the convective flux density vector on a cell face is computed with the Jameson scheme (Jameson et al., 1981). The artificial viscosity includes a second-order dissipation term $D_{2}$ and a fourth-order dissipation term $D_{4}$, which involve two tunable parameters $k^{(2)}$ and $k^{(4)}$.

The viscous terms are discretized by a second-order space-centered scheme. For the turbulence transport equations, the upwind Roe scheme (Roe, 1981) is used to obtain a more robust method. The second-order accuracy is obtained by introducing a flux-limited dissipation (Tatsumi et al., 1995).

\subsection{The low Mach number preconditioner}

For low Mach number applications, a well-known problem concerns the stiffness on the solution convergence. In this situation, the dominance of convection terms renders the system stiff and compressible solvers converge slowly. To overcome this difficulty, a preconditioning method is necessary. The physical acoustic waves are replaced by pseudo-acoustic modes that are much closer to the advective velocity, reducing the stiffness and enhancing the convergence. The method is based on the modification of the derivative term by a premultiplication with a suitable preconditioning matrix. In order to simplify the formulation, we present below the one-dimensional formulation. With the primitive variables $W=(P, u, e, \alpha)$ the preconditioned Euler equations can be expressed as:

$$
P_{e}^{-1} \frac{\partial W}{\partial t}+A_{e} \frac{\partial W}{\partial x}=0
$$

We use the preconditioning matrix based on the Turkel approach (Guillard and Viozat, 1999; Turkel, 1987) : 


$$
P_{e}=\left[\begin{array}{cccc}
\beta^{2} & 0 & 0 & 0 \\
0 & 1 & 0 & 0 \\
0 & 0 & 1 & 0 \\
0 & 0 & 0 & 1
\end{array}\right] \quad ; \quad A_{e}=\left[\begin{array}{cccc}
u & \rho c^{2} & 0 & 0 \\
1 / \rho & u & 0 & 0 \\
0 & P / \rho & u & 0 \\
0 & -K & 0 & u
\end{array}\right]
$$

$\beta$ is a parameter on the order of the Mach number. In our study, we have chosen the form given by Choi and Merkle (Choi and Merkle, 1993):

$$
\beta^{2}=\min \left[\max \left(M^{2}, \theta M_{\infty}^{2}\right), 1\right]
$$

This form implies that there is no preconditioning used in transonic and supersonic flow regions (in the mixture). When $\beta^{2}=1$, the preconditioning matrix becomes the identity matrix and the system returns to its classical non preconditioned form. Moreover, for a very small flow velocity, $\beta^{2}$ is not allowed to be less than a given percentage of the freestream velocity, determined by the coefficient $\theta$.

The eigenvalues of the preconditioned system are:

$$
u ; u \quad ; \quad \lambda_{ \pm}=\frac{1}{2}\left[u\left(1+\beta^{2}\right) \pm \sqrt{\left(\beta^{2}-1\right)^{2} u^{2}+4 \beta^{2} c^{2}}\right]
$$

For the variables $w=[\rho, \rho u, \rho E, \alpha]$, the corresponding form is:

$$
P_{c}^{-1} \frac{\partial w}{\partial t}+A_{c} \frac{\partial w}{\partial x}=0
$$

where the preconditioning matrix $P_{c}^{-1}=\frac{\partial w}{\partial W} P_{e}^{-1} \frac{\partial W}{\partial w}$ and $A_{c}$ is the Jacobian matrix of the convective fluxes. Expressions of matrices are given in appendix A.

The preconditioned matrix can be written as $P_{c}^{-1}=I_{d}+\frac{\left(1-\beta^{2}\right)}{\beta^{2}} \times M$ where $I_{d}$ is the identity matrix and the expression of the matrix $M$ is given in Appendix B. 
The matrix $M$ is idempotent and the inverse matrix $P_{c}$ can be easily computed: $P_{c}=I_{d}+\left(\beta^{2}-1\right) \times M$.

In the mixture area (compressible zone), $\beta^{2}=1$ and $P_{c}=I_{d}$ therefore the classical compressible system is integrated.

\subsection{Temporal discretization}

Time integration is achieved using the dual time stepping approach and a lowcost implicit method consisting in solving, at each time step, a system of equations arising from the linearization of a fully implicit scheme. The derivative with respect to the physical time is discretized by a second-order formula. The main advantage of this method is that the storage of the Jacobian matrix is completely eliminated, which leads to a low-storage algorithm (Goncalves and Patella, 2009).

With the preconditioned method, the dissipation matrices are modified and the preconditioned matrix $P_{c}^{-1}$ remains in the formulation. By judiciously exploiting the idempotence propriety of the matrix $M$, it is possible to preserve a low-cost system where matrix operations and matrix-vector products can be easily computed.

For the turbulence transport equations, the diffusive flux Jacobian matrix is replaced by its spectral radius. The source term needs special treatment (Merci et al., 2000). Only the negative part of the source term Jacobian matrix is considered and replaced by its spectral radius. The system obtained is solved with a line-alternated Jacobi relaxation algorithm.

\subsection{Inlet and outlet boundary conditions}

The numerical treatment of the boundary conditions is based on the use of the preconditioned characteristic relations of Euler equations. The number of variables 
to impose at boundaries is given by the number of characteristics directed into the domain of interest. The characteristic relations obtained for the preconditioned system, in two-dimensional flows, are:

$$
\begin{aligned}
-c^{2}\left(\rho^{c}-\rho^{s}\right)+\left(P^{c}-P^{s}\right) & =0 \\
V_{t}^{c}-V_{t}^{s} & =0 \\
\rho\left(\alpha^{c}-\alpha^{s}\right)-K\left(\rho^{c}-\rho^{s}\right) & =0 \\
\left(\lambda_{+}-V_{n}\right)\left(P^{c}-P^{s}\right)+\rho \beta^{2} c^{2}\left(V_{n}^{c}-V_{n}^{s}\right) & =0 \\
\left(\lambda_{-}-V_{n}\right)\left(P^{c}-P^{s}\right)+\rho \beta^{2} c^{2}\left(V_{n}^{c}-V_{n}^{s}\right) & =0
\end{aligned}
$$

The variables with superscript $c$ denote the variables to be computed at the boundary. Variables with superscript $s$ denote the variables obtained by the current numerical scheme. $V_{t}$ and $V_{n}$ are the tangential and the normal component of the mean velocity, respectively.

At inflow, we impose the stagnation pressure $P_{i}$, the stagnation temperature $T_{i}$, the direction of the velocity and the initial values of the void ratio. The pressure is evaluated with the relation (23) and all variables can be evaluated at the boundary. At outflow, the static pressure is imposed. The conservative variables are computed with four characteristic relations (19)-(22).

\section{2D inviscid problems}

For these cases, computations were performed solving the one-fluid compressible Euler equations.

\subsection{Underwater explosion with cavitation}

The cylindrical underwater explosion near a flat free surface is considered. A similar case has been studied in (Liu et al., 2001; Xie et al., 2007). The initial conditions 
are given as follows. A high-pressured gas cylinder with a radius of $0.5 \mathrm{~m}$ is located at the origin $(0,0)$ and the initial flow parameters for explosive gas, water and air are: $\rho_{g}=1270 \mathrm{~kg} / \mathrm{m}^{3}, P_{g}=8290 \mathrm{bar}, u_{g}=v_{g}=0.0$. and $\gamma_{g}=2 ; \rho_{l}=1150 \mathrm{~kg} / \mathrm{m}^{3}, P_{l}=1$ bar, $u_{l}=v_{l}=0$ and $\gamma_{l}=2.35 ; P_{\text {pair }}=1$ bar, $\rho_{\text {air }}=1 \mathrm{~kg} / \mathrm{m}^{3}, u_{\text {air }}=v_{\text {air }}=0$ and $\gamma_{\text {air }}=1.4$. The stiffened gas parameters are given in Table 1 . The constant $c_{\text {baro }}$ is set to $0.92 \mathrm{~m} / \mathrm{s}$ and $C_{d e s}=0$. The vapor pressure $P_{v a p}=51000 \mathrm{~Pa}$. The computational domain is a rectangular region with $x \times y \in[-6,6] \times[6,6]$ and the free surface is located at the straight line $y=2 \mathrm{~m}$ (see Figure 1 ). An uniform grid composed by $800 \times 800$ nodes is distributed in computational domain. The top and bottom boundary conditions are walls. On other frontiers, variables are extrapolated. The time step is set to $10^{-6} \mathrm{~s}$.

Figure 2 illustrates a series of pressure contours as time progresses where the shock and free surface interaction and cavitation evolution are clearly observed. At $t=1.0$ ms (Fig. 2(a)), the initial shock generated by the explosion has impacted the free surface and reflected with strong rarefaction waves (Prandtl-Meyer rarefaction waves) while a transmitted shock is propagating into the air medium. This transmitted shock cannot be discerned in figures because it is much weaker than the reflected rarefaction waves due to the much lower acoustic impedance of the air medium. The Prandtl-Meyer rarefaction waves are propagating in the opposite directions along the water-air interface and cause the water pressure just below the free surface to drop very rapidly. With the decrease of the water pressure, a small cavitation pocket appears just below the free surface as shown in Fig. 2(b) at $t=1.5 \mathrm{~ms}$. Figs. 2(c) and $2(\mathrm{~d})$ show the growth of the cavitation pocket below the free surface as the propagation of the rarefaction waves goes on. Overall, results are in good agreement with those presented in (Xie et al., 2007). 


\subsection{Compression of a vapour bubble}

Our code is tested on the case of a bubble compression with a collapsing process. Similar cases were simulated in (Faccanoni, 2008; Faccanoni et al., 2012). We consider a $1 \mathrm{~m}$ side-length square domain discretized over a $400 \times 400$ cells mesh. A vapour bubble is located on the center of the domain and is surrounded by liquid phase (Figure 3). The radius of the bubble is initially $0.2 \mathrm{~m}$. The initial temperature $T_{0}$ is $354 \mathrm{~K}$ and the fluid is initially at rest in the whole domain. Both phases are supposed to be at saturation at $t=0$ and the vapour pressure is $P_{\text {sat }}\left(T_{0}\right)=51000$ Pa. We suppose the left boundary to be a piston and the velocity $u_{p}$ is set to 30 $\mathrm{m} / \mathrm{s}$. Other boundary conditions are reflective walls. The stiffened gas parameters are those used in the previous cases. During the collapse, high pressures are reached and the mass transfer model is not activated. The vapour phase is considered to be non condensable. The time step used for the simulation is $10^{-7} \mathrm{~s}$.

Figure 4 shows the evolution of the void ratio (iso-lines) for time varying from $t=$ $0 \mathrm{~ms}$ to $\mathrm{t}=8 \mathrm{~ms}$. The moving piston generates a pressure wave that compresses the gas. Due to the pressure difference between back and forth of pressure waves, the bubble is asymmetrically contracted with concave shape. As time moves on, the bubble becomes kidney shaped and spreads laterally in the process. This change in shape is driven by vorticity generated at the edge of the bubble due to the passage of the wave which induces a jet of water along the axis of flow symmetry. When this jet impinges on the water at the downstream edge of the bubble, the bubble forms a pair of distinct vortical structures (time $t=7 \mathrm{~ms}$ and after).

Such physical phenomena were described in the case of shock-bubble interactions. According to the sign of the difference of acoustic impedance of pure phases $d R=$ 
$\rho_{v} c_{v}-\rho_{l} c_{l}$, two scenarii are depicted. In our case, $d R<0$ and this situation is commonly referred to as divergent geometry (Ranjan et al., 2011). After several shockpassage times, the features observed in the flow field are dominated by the vortical motion. The vorticity-generation mechanism in the absence of dissipative effects is due to the baroclinic term and the noncollinearity of the local pressure and density gradients.

$$
\frac{D \omega}{D t}=\frac{1}{\rho^{2}} \operatorname{grad} P \wedge \overrightarrow{\operatorname{grad}} \rho
$$

The iso-surfaces of the baroclinic term modulus are plotted in Figure 5 (the unit is $\left.1 / s^{2}\right)$ at two times. At time $\mathrm{t}=0.5 \mathrm{~ms}$, the maximum misalignment is at the diametral vertical plane, and the maximum vorticity is deposited at this location. At time $\mathrm{t}=1.5 \mathrm{~ms}$, the maximum values of vorticity have moved, creating the asymmetric shape.

The mixture temperature profile in a horizontal cutting plane $y=0.5 \mathrm{~m}$ is plotted in Figure 6 at different times from $t=0.5 \mathrm{~ms}$ to $t=4 \mathrm{~ms}$ (a logarithmic scale is used for the temperature). A temperature increase up to $3500 \mathrm{~K}$ is observed at time $\mathrm{t}=2 \mathrm{~ms}$. After this time, the temperature decreases to reach $360 \mathrm{~K}$ at the end of the simulation. In comparison with other simulations (Nourgaliev et al., 2006; Muller et al., 2010), the maximal temperature value is not incoherent. The lack of viscosity and heat conduction in the present simulations may significantly affect the extremal values of thermodynamic quantities. Moreover, it was observed that the grid refinement could lead to an excessive smearing of interfaces and numerical instabilities.

\subsection{Compression of four vapour bubbles}

The same test case is considered with four bubbles. Similar cases were simulated in (Faccanoni, 2008; Faccanoni et al., 2010). Four vapour bubbles are surrounded 
by liquid phase located in the domain as illustrated in Figure 7 . The radius of each bubble is initially $0.1 \mathrm{~m}$. The initial temperature $T_{0}$ is $354 \mathrm{~K}$ and the fluid is initially at rest in the whole domain. Both phases are supposed to be at saturation at $t=0$ and the vapour pressure is $P_{\text {sat }}\left(T_{0}\right)=51000 \mathrm{~Pa}$. We suppose the left boundary to be a piston and the velocity $u_{p}$ is set to $30 \mathrm{~m} / \mathrm{s}$. Other boundary conditions are reflective walls. The stiffened gas parameters are those used in the previous cases. The time step used for the simulation is $10^{-7} \mathrm{~s}$.

Figure 8 shows the iso-lines of the volume fraction for time varying from $t=0$ $\mathrm{ms}$ to $t=8 \mathrm{~ms}$. From the top to the bottom the following sequence can be seen: the moving piston generates a pressure wave that compresses the vapour. As the pressure increases the vapour starts to condense and the bubble shrinks. Due to the pressure difference between back and forth of the compression wave, the bubbles are asymmetrically contracted as observed in the previous case. At this time, a sort of microjet is formed and eventually it impinges on the rear surface of the bubble with reflection. Bubble is gradually shrunken, pressure in the bubble reaches maximum value around 220 bar and a rebound wave occurs. This wave propagates to liquid region and the bubble is expanded with time. The bubble collapsing behaviour with rebound is well simulated with the solver.

\section{2D turbulent Venturi cases}

\subsection{Experimental data}

Both the Venturi geometries were tested in the cavitation tunnel of the CREMHyG (Centre d'Essais de Machines Hydrauliques de Grenoble). The first one is characterized by a divergence angle of $4^{\circ}$ and the second by a divergence angle of $8^{\circ}$, illustrated 
in Fig. 9. The pressure $P_{\text {inlet }}$ was lowered until the desired cavitation number allowing two specific behaviours of the sheet cavity. The cavitation number in the inlet section is defined as: $\sigma_{\text {inlet }}=\frac{P_{\text {inlet }}-P_{\text {vap }}}{0.5 \rho U_{\text {inlet }}^{2}}$, where $P_{\text {vap }}$ is the vapour pressure at $20^{\circ} \mathrm{C}$ and $P_{\text {inlet }}, U_{\text {inlet }}$ are the pressure and velocity respectively at the reference section upstream of the Venturi. The geometrical data and flow configurations are given in Table 2.

With these previous parameters and according to experimental observations (Barre et al., 2009; Patella et al., 2006), cavitation sheets developed from the Venturi throat. The obtained cavity length is ranging from $70 \mathrm{~mm}$ to $85 \mathrm{~mm}$ for case 1 and having a relatively stable aspect (see Figure 10). The attached cavity length corresponding to the end of the re-entrant jet is around $30 \mathrm{~mm}$. For this case, no periodic cycles with large shedding were observed.

For case 2, a typical self-oscillation behaviour was observed with quasi-periodic vapour clouds shedding and the maximum cavity length (before the break-off of the cavity) was $45 \pm 5 \mathrm{~mm}$. Figure 10 shows an instantaneous photograph of the cavity with a large structure shedding. The cloud shedding frequency was about 45 Hz. The divergent part was equipped with eight probe holes to take various measurements such as the instantaneous pressure, local void ratio and velocity in the sheet cavity. Specific probe locations are presented in Figure 9 for the two tested cases. The relative uncertainty on the void ratio measurement was estimated at roughly 15\% (Barre et al., 2009).

\subsection{Numerical data, parameters and meshes}

Both grids are a H-type topology. A special contraction of the mesh is applied in the main flow direction just after the throat to better simulate the two-phase flow 
area (Fig. 11).

The parameter $c_{\text {baro }}$ was set to $0.92 \mathrm{~m} / \mathrm{s}$ as previously. The parameters of the stiffened gas EOS are given in Table 3.

For the non cavitating regime, computations are started from an uniform flow-field using a local time step. For the unsteady cavitating regime, computations are performed with the dual time stepping method and are started from the non cavitating numerical solution. The numerical parameters are given in Table 4.

All numerical values are obtained by a time-averaged treatment on a physical time around $2.5 \mathrm{~s}$. For case 2, a direct Fourier transformation (DFT) of the vapour volume signal was performed to evaluate the frequency.

\subsection{Case 1: aperiodic oscillating partial cavity}

Five calculations were performed by varying the parameter $C_{d e s}$ from 0 to 100 $(0,0.1,1,10,100)$. The goal was to obtain a time-averaged cavitation sheet whose length varied between $70-85 \mathrm{~mm}$ with a re-entrant jet. The time of simulation is around 4 s. For all simulations, the inlet cavitation number varied between 0.59 and 0.6 .

A qualitative description of the dynamic of cavity sheets is proposed with the plotting of the contours of the density gradient modulus (Schlieren-like visualizations) and the iso-lines of the Q-criterion. Positive values of the Q-criterion, defined as the second invariant of the velocity gradient tensor $\frac{\partial u_{i}}{\partial x_{j}}$ (Hunt et al., 1988),

$$
Q=\frac{1}{2}\left[\left(\frac{\partial u_{i}}{\partial x_{i}}\right)^{2}-\frac{\partial u_{i}}{\partial x_{j}} \frac{\partial u_{j}}{\partial x_{i}}\right]
$$


are used to identify vortices and local rotational areas. A dimensionless quantity is built with the inlet velocity and the reference length. Iso-lines levels vary between 0.01 and 1 .

Figure 12 illustrates both contours of density gradients (on the left) and the dimensionless Q-criterion (on the right), at two instants, obtained with the value $C_{d e s}=1$. The cavity interface is well exhibited by the density gradient contours. Around the abscissa $x=0.35 \mathrm{~m}$, we can observe the attached cavity closure and downstream the fluctuating recirculation with two-phase structures shedding. The generated shear layer with vortical clouds of cavitation is clearly shown with the Q-criterion. For this case, the wall jet is probably not intense enough to break the sheet and to induce cyclic clouds shedding. It is a transitional behaviour between a stable cavity and a self-oscillating one.

\subsubsection{Velocity and void ratio profiles}

Local analyses concern void ratio and velocity profile comparisons inside the cavity. The experimental void ratio and velocity profiles are obtained for five stations by a double optical probe (Fig. 9).

Figure 13 shows the longitudinal velocity profiles (on the left) for the experiments and the five computations. The overall agreement seems good between the experimental data and the simulations. For stations 1 and 2, no re-entrant jet phenomena occurs in the experiment. With the value of $C_{d e s}=100$, the flow is near to separate at station 2. Further downstream, for stations 3, 4 and 5, experimental observation indicates a recirculating behaviour with a re-entrant jet extending roughly through half the sheet thickness. This recirculating behaviour with a re-entrant jet is well 
simulated by all computations. At station 3, the intensity and the thickness of the recirculating area are in better agreement with the experimental data when the destruction term is activated. The influence of $C_{d e s}$ is weak, a small discrepancy being noticeable on the thickness of the recirculation. At station 4, all simulations provided a similar result, in correct agreement with the measurement. At station 5 , the intensity of the recirculation area is under-estimated by all simulations.

Figure 13 illustrates experimental and numerical results concerning the void ratio profiles (on the right). For the two first stations, close to the throat, the vaporization phenomenon is clearly represented. This is a relatively strong effect, and the void ratio value can reach $98 \%$. For all computations, the cavity thickness is very well estimated. At station 3, the re-entrant jet becomes noticeable, as observed before in the velocity field analyses. The decrease of the void ratio values inside the cavity is different according to the model. Without the destruction term, the maximum value is over-predicted ( $80 \%$ instead of $60 \%$ for the experimental data) and the decrease up to the wall is monotonic. The numerical wall value is also over-predicted ( $60 \%$ instead of $30 \%$ in the experimental data). When the destruction term is activated, the maximum value is in better agreement with the experimental data. Yet, we observed a non monotonic behaviour with a decrease on the half top of the cavity following by an increase up to the wall. At station 4, the shape of the void ratio profile is similar for all simulations with an over-prediction of the maximal value. At the last station, excepted without the destruction term, the void ratio values are under-estimated.

To conclude, the results given by the new model are in good agreement with the experimental data. The influence of the parameter $C_{d e s}$ seems weak for the timeaveraged profiles. 


\subsubsection{Wall pressure and RMS fluctuations}

The time-averaged wall pressure distribution is plotted in Fig. 14 versus the distance $x-x_{\text {inlet }}$. The first five data are located inside the cavity (where the void ratio and velocity profiles are measured). For all computations, the pressure remains at an almost constant value $P_{v a p}$ in the cavity. Downstream, all computations provided a similar result. Only small discrepancies are noticeable for the cavity length.

The Root Mean Square (RMS) wall pressure fluctuations are plotted in Fig. 15 versus the distance $x-x_{\text {inlet }}$. The pressure fluctuation is divided by the time-averaged pressure Pav. For all computations, the statistical treatment was performed on a simulation time of $1 \mathrm{~s}$. Experimental data indicate an augmentation of pressure fluctuation at the end of the cavity sheet, with a peak located at the fifth station.

All simulations predicted a large peak of fluctuations at the end of the cavity, between the fourth and the fifth station. The peak position and the intensity do not vary between simulations including the destruction term. Downstream, from station 6 to 9 , the level of fluctuations is well computed with all the simulations including the destruction term. Without this term, the peak of fluctuations is more extended and, downstream, the plateau of fluctuations is largely over-estimated.

The importance of the destruction term through the parameter $C_{d e s}$ is clearly illustrated. From 0.1 to 100 , the value of $C_{\text {des }}$ has a very weak influence on the RMS pressure fluctuations.

\subsubsection{Comparison with a barotropic model}

In relation to previous numerical studies developed on the same Venturi geometry using a barotropic 3-equation model (Goncalves, 2011; Goncalves and Decaix, 2012), the new results obtained with $C_{d e s}=1$ are quite similar. In Fig. 16 are plotted 
the time-averaged void ratio and velocity profiles at station 3 and 4 , and the wall pressure evolution. The velocity profiles are very similar. At station 4, the void ratio profile is better predicted by the 4-equation model. The time-averaged wall pressure evolution is quite identical. About the RMS wall pressure fluctuations, a small discrepancy is noticeable about the peak location.

In conclusion, for this partial cavity, the 4-equation model does not allow to clearly improve the previous simulations obtained with a barotropic model.

\subsection{Case 2: periodic oscillating partial cavity}

Different calculations were performed by varying the value of the parameter $C_{d e s}$, summarized in Table 5. The goal was to obtain a periodic cavitation sheet with a frequency close to $45 \mathrm{~Hz}$. The simulation time is around $3 \mathrm{~s}$.

About the inlet cavitation number, for all simulations it varied between 2.18 and 2.25 (the experimental value was $2.15 \pm 0.06$ ). All calculations captured a periodic self-oscillating cavity but discrepancies are noticeable according to the value of the parameter $C_{d e s}$. Between 1 and 20, the influence of $C_{d e s}$ is strong on the frequency. Up to $C_{d e s}=6$, simulations provided a quasi cyclic phenomenon with a frequency around $45 \mathrm{~Hz}$. For higher values, the frequency decreases as the value increases. With $C_{d e s}=20$, the frequency is around $7 \mathrm{~Hz}$. The dynamic of the sheet cavitation driven by the re-entrant jet is clearly influenced by the destruction term.

Figure 17 illustrates both contours of density gradients modulus (on the left) and the dimensionless Q-criterion (on the right), during one period, obtained with $C_{d e s}=1$. Q-criterion iso-lines levels vary between 0.01 and 0.1 . We can observe the selfoscillating cavity cycle: the cavity attached to the wall grows up to the generation 
of a re-entrant jet. This one flows upstream along the wall and leads to the break-off of the downstream part of the cavity. The resulting cloud of vapour is then carried away by the main stream, until it enters a higher-pressure zone and collapses. The remaining part of the attached cavitation sheet re-expands and a new cycle starts.

\subsubsection{Velocity and void ratio profiles, wall pressure evolution}

Figure 18 shows the evolution of the longitudinal velocity (on the left) and void ratio time-averaged profiles (on the right) for the experiments and computations with $C_{d e s}=0,1,4$ and 6 . For the velocity profiles, experimental data are given for the first two stations. At station 1, all calculations provided a similar result in good agreement with the experimental data. At station 2, the intensity of the recirculation is under-predicted with the model without the destruction term. When this term is activated, the recirculating area is largely reduced. With $C_{d e s}=6$, the time-averaged velocity is no more negative.

About the void ratio profiles, experimental values are weak, even at station 1. For this station, all simulations largely over-predicted the void ratio, with a factor of 2 . At stations 2 and 3, the void ratio is still over-predicted. The influence of the parameter $C_{\text {des }}$ can be well observed: higher is the value, smaller is the void ratio value.

The time-averaged dimensionless wall pressure distribution is plotted in Figure 19 versus the distance $x-x_{\text {inlet }}$. Results obtained with all models are in correct agreement with the experimental data. Discrepancies are noticeable near the throat at station 1. Higher is the $C_{d e s}$ value, more the dimensionless pressure value is overestimated. 


\subsubsection{Comparison with a barotropic model}

In comparison with previous simulations on the same Venturi geometry using a barotropic 3-equation model (Goncalves, 2011; Goncalves and Decaix, 2012), the new results provided a shedding phenomenon in better agreement with the experimental data.

With the barotropic model, we simulated a periodic phenomenon with a frequency close to $46 \mathrm{~Hz}$. The unsteady behaviour of the cavity was clearly different, as illustrated by the density gradient and the Q-criterion in Figure 20. We can observe a small attached cavity, and downstream the fluctuating recirculation with two-phase structures shedding. These shedding are not extended and are rapidly eliminated. In comparison with the experimental visualizations and the 4-equation simulations, the attached cavity and the clouds shedding are largely under-estimated.

In Figure 21 are plotted the time-averaged velocity and void ratio profiles obtained with the 3 -equation model and the 4 -equation model with $C_{d e s}=1$. Large discrepancies are highlighted on the void ratio profiles. Near the throat, the void ratio is largely over-estimated using the 3-equation model, whereas at the last station, the averaged value is null. As commented previously, the cloud shedding is not enough developed. On the contrary, it is more intense with the 4-equation model.

Discrepancies on the recirculating area and the re-entrant jet are noticeable at station 2 . With the 4-equation model, the recirculating area is largely under-predicted. Finally, the time-averaged dimensionless wall pressure is presented in the same figure. Both models provided a result in correct agreement with the experimental data. 


\section{Conclusion}

In this paper, a 4-equation model was developed to study cavitation pockets which can occur in a variety of practical cases. This model is composed by three conservations law for mixture quantities (mass, momentum, total energy) and an additional transport equation for the vapour volume fraction, where mass transfer rate due to cavitation is modelled. The new formulation is based on the assumption of proportionality of the mass transfer with the divergence of velocity and includes a destruction term involving a tunable parameter. The model has been implemented in a compressible RANS solver including a low Mach number preconditioning algorithm and has been applied for the simulation of various unsteady cavitation problems (for both inviscid and turbulent cases).

First validations on inviscid cases shown the ability of the model to simulate the cavitation development in an underwater explosion and the asymmetrical bubble collapse by a pressure wave. Secondly, RANS simulations were performed to predict the unsteady behaviour of partial cavities developing along Venturi geometries (quasi-stable and unstable cases). Numerical results obtained from the new model have been validated against experimental data and the influence of the destruction term was investigated. Moreover, the new model was compared with a barotropic 3equation model previously developed in our team. These test-cases lead to different concluding remark:

- For the quasi-stable cavity, a good agreement between numerical results and experimental data has been obtained with the model. The destruction term weakly influenced the time-averaged profiles. On the contrary, its activation was determinant for the RMS pressure fluctuations prediction. In comparison with the 3-equation simulations, both models provided similar results. The new model did not clearly 
improve the previous simulations.

- For the periodic self-oscillating cavity, the overall results suggest that the model reasonably simulated the sheet dynamic. The influence of the destruction term thought the tunable parameter $C_{\text {des }}$ was strong with regard to the phenomenon frequency. Only values smaller than 6 allowed to predict the good frequency. In comparison with the 3-equation model, the two-phase structures shedding was more intense and in better agreement with the experimental data.

Additional works are in progress to pursue comparative analyses between turbulence and cavitation models and to optimize the calibration of the model parameters.

\section{Appendix}

Appendix A: Expression of matrices $\frac{\partial w}{\partial W}$ and $\frac{\partial W}{\partial w}$

Starting from the differential of $\rho e$ expressed with variables $(P, \rho, \alpha)$

$$
\begin{aligned}
d(\rho e) & =A d \rho+B d P+C d \alpha \\
A & =\left(\frac{\partial \rho e}{\partial \rho}\right)_{P, \alpha}=\alpha\left(q_{v}-q_{l}\right)\left(\frac{\partial \rho_{v}}{\partial \rho}\right)_{\alpha} \\
B & =\left(\frac{\partial \rho e}{\partial P}\right)_{\rho, \alpha}=\frac{\alpha}{\gamma_{v}-1}+\frac{1-\alpha}{\gamma_{l}-1}=\frac{1}{\gamma-1} \\
C & =\left(\frac{\partial \rho e}{\partial \alpha}\right)_{\rho, P}=\rho_{v}\left(e_{v}-q_{v}\right)-\rho_{l}\left(e_{l}-q_{l}\right)+\rho_{I}\left(q_{v}-q_{l}\right)
\end{aligned}
$$

From the conservation law for the vapour mass, we have

$$
\begin{aligned}
\frac{d \rho_{v}}{d t} & =\frac{1}{\alpha}\left(\dot{m}-\rho_{v} \frac{d \alpha}{d t}\right)-\rho_{v} \frac{\partial u}{\partial x} \\
& =\frac{\rho_{I}}{\alpha}\left(1-\frac{\rho_{v}}{\rho_{I}}\right) \frac{d \alpha}{d t}+\frac{\rho_{I}}{\rho}\left(\frac{K}{\alpha}+\frac{\rho_{v}}{\rho_{I}}\right) \frac{d \rho}{d t}
\end{aligned}
$$

We introduce the speed of sound $\tilde{c}$ defined as

$$
\frac{1}{\tilde{c}^{2}}=\frac{1-\alpha}{c_{l}^{2}}+\frac{\alpha}{c_{v}^{2}}
$$


The expression of the quantity $A$ is

$$
\begin{aligned}
\left(\frac{\partial \rho_{v}}{\partial \rho}\right)_{\alpha} & =\frac{\rho_{I}}{\rho}\left(\frac{K}{\alpha}+\frac{\rho_{v}}{\rho_{I}}\right)=\frac{\tilde{c}^{2}}{c_{v}^{2}} \\
A & =\alpha\left(q_{v}-q_{l}\right) \frac{\tilde{c}^{2}}{c_{v}^{2}}
\end{aligned}
$$

The differentials of $u, e$ and $P$ expressed with conservatives variables and the void ratio are

$$
\begin{aligned}
d u & =\frac{1}{\rho} d(\rho u)-\frac{u}{\rho} d \rho \\
\rho d e & =\left(\frac{u^{2}}{2}-e\right) d \rho-\rho u d(\rho u)+d(\rho E) \\
B d P & =d(\rho e)-A d \rho-C d \alpha=d(\rho E)-u d(\rho u)+\left(\frac{u^{2}}{2}-A\right) d \rho-C d \alpha
\end{aligned}
$$

Moreover, using the equation for the pressure, we have

$$
\frac{1}{\tilde{c}^{2}} d P=d \rho+\left(\rho_{l}-\rho_{v}\right) d \alpha
$$

We deduce the differential of the void ratio

$$
\left[B \tilde{c}^{2}\left(\rho_{l}-\rho_{v}\right)+C\right] d \alpha=d(\rho E)-u d(\rho u)+\left(\frac{u^{2}}{2}-A-B \tilde{c}^{2}\right) d \rho
$$

Finally, the expression of the matrix $\frac{\partial W}{\partial w}$ is

$$
\frac{\partial W}{\partial w}=\left[\begin{array}{cccc}
(\gamma-1)\left(\frac{u^{2}}{2}-A\right) & -(\gamma-1) u & (\gamma-1) & -(\gamma-1) C \\
-u / \rho & 1 / \rho & 0 & 0 \\
\frac{u^{2}-E}{\rho} & -\frac{u}{\rho} & \frac{1}{\rho} & 0 \\
\frac{\frac{u^{2}}{2}-A-B \tilde{c}^{2}}{B \tilde{c}^{2}\left(\rho_{l}-\rho_{v}\right)+C} & \frac{u}{B \tilde{c}^{2}\left(\rho_{l}-\rho_{v}\right)+C} & \frac{1}{B \tilde{c}^{2}\left(\rho_{l}-\rho_{v}\right)+C} & 1
\end{array}\right]
$$


We introduce the quantities $F$ and $G$

$$
\begin{aligned}
F & =\frac{\tilde{c}^{2}}{\gamma-1}\left(\rho_{l}-\rho_{v}\right)+C \\
G & =(e-A)(F+C)-C \frac{\tilde{c}^{2}}{\gamma-1}
\end{aligned}
$$

The expression of the inverse matrix $\frac{\partial w}{\partial W}$ is

$$
\frac{\partial W}{\partial w}=\left[\begin{array}{cccc}
\frac{F}{G(\gamma-1)} & 0 & -\frac{\rho(F+C)}{G} & \frac{F C}{G} \\
\frac{F}{G(\gamma-1)} u & \rho & -\frac{\rho(F+C)}{G} u & \frac{F C}{G} u \\
\frac{F}{G(\gamma-1)} E & \rho u & -\frac{\rho}{G}\left[(F+C)\left(\frac{u^{2}}{2}+A\right)+C \frac{\tilde{c}^{2}}{\gamma-1}\right] & \frac{F C}{G} E \\
-\frac{e-A-\frac{\tilde{c}^{2}}{\gamma-1}}{G(\gamma-1)} & 0 & -\frac{\rho \tilde{c}^{2}}{G(\gamma-1)} & \frac{F(e-A}{G}
\end{array}\right]
$$

Appendix B: Preconditioning matrix

The preconditioned matrix can be written as $P_{c}^{-1}=I_{d}+\frac{\left(1-\beta^{2}\right)}{\beta^{2}} \times M$. We introduce the quantity $J=\frac{1-\frac{\gamma-1}{\tilde{c}^{2}}(e-A)}{\left(\rho_{l}-\rho_{v}\right)+C\left(\frac{\gamma-1}{\tilde{c}^{2}}\right)}$. The expression of the matrix $M$ is

$$
M=\frac{F}{G} \times\left[\begin{array}{cccc}
\frac{u^{2}}{2}-A & -u & 1 & -C \\
\left(\frac{u^{2}}{2}-A\right) u & -u^{2} & u & -C u \\
\left(\frac{u^{2}}{2}-A\right) E & -u E & E & -C E \\
\left(\frac{u^{2}}{2}-A\right) J & -u J & J & -C J
\end{array}\right]
$$




\section{References}

Barre, S., Rolland, J., Boitel, G., Goncalves, E., Patella, R. F., 2009. Experiments and modelling of cavitating flows in Venturi: attached sheet cavitation. European Journal of Mechanics B/Fluids 28, 444-464.

Chen, Y., Lu, C., Wu, L., 2006. Modelling and computation of unsteady turbulent cavitation flows. Journal of Hydrodynamics 18 (5), 559-566.

Choi, Y., Merkle, C., 1993. The application of preconditioning to viscous flows. Journal of Computational Physics 105 (2), 207-223.

Coutier-Delgosha, O., Fortes-Patella, R., Reboud, J., 2002. Simulation of unsteady cavitation with a two-equation turbulence model including compressibility effects. Journal of Turbulence 3 (58).

Decaix, J., Goncalves, E., 2013. Compressible effects modelling in turbulent cavitating flows. European J. of Mechanics B/Fluids 39, 11-31.

Delannoy, Y., Kueny, J., 1990. Two phase flow approach in unsteady cavitation modelling. In: Cavitation and Multiphase Flow Forum, ASME-FED, vol. 98, pp.153158.

Faccanoni, G., 2008. Etude d'un modèle fin de changement de phase liquide-vapeur. contribution à l'étude de la crise d'ébullition. Ph.D. thesis, Ecole Polytechnique, France. online http://pastel.paristech.org/4785/.

Faccanoni, G., Kokh, S., Allaire, G., 2010. Approximation of liquid-vapor phase transition for compressible fluids with tabulated eos. C. R. Acad. Sci. Paris Ser. I (348), 473-478. 
Faccanoni, G., Kokh, S., Allaire, G., 2012. Modelling and simulation of liquid-vapor phase transition in compressible flows based on thermodynamical equilibrium. Math. Modelling and Numerical Analysis (46), 1029-1054.

Goncalves, E., 2011. Numerical study of unsteady turbulent cavitating flows. European Journal of Mechanics B/Fluids 30 (1), 26-40.

Goncalves, E., 2012. Simulation of rarefaction problems with phase transition. In: $8^{\text {th }}$ International Symposium on Cavitation CAV2012, Singapore. http://cav2012.sg/proceedings/index.html.

Goncalves, E., 2013. Numerical study of expansion tube problems: Toward the simulation of cavitation. Computers \& Fluids 72, 1-19.

Goncalves, E., Decaix, J., 2012. Wall model and mesh influence study for partial cavities. European Journal of Mechanics B/Fluids 31 (1), 12-29.

Goncalves, E., Patella, R. F., 2009. Numerical simulation of cavitating flows with homogeneous models. Computers \& Fluids 38 (9), 1682-1696.

Goncalves, E., Patella, R. F., 2011. Constraints on equation of state for cavitating flows with thermodynamic effects. Applied Math. and Computation 217, 50955102.

Guillard, H., Viozat, C., 1999. On the behaviour of upwind schemes in the low Mach number limit. Computers \& Fluids 28 (1), 63-86.

Helluy, P., Seguin, N., 2006. Relaxation models of phase transition flows. Mathematical Modelling and Numerical Analysis 40 (2), 331-352. 
Hunt, J., Wray, C., Moin, P., 1988. Eddies, streams, and convergence zones in turbulent flows. Tech. rep., Center for Turbulence Research, CTR-S88.

Ihm, S., Kim, C., 2008. Computations of homogeneous-equilibrium two-phase flows with accurate and efficient shock-stable schemes. AIAA Journal 46 (12), 30123037.

Ishii, C., Hibiki, T., 2006. Thermo-fluid dynamics of two-phase flow. Springer.

Jameson, A., Schmidt, W., Turkel, E., 1981. Numerical solution of the Euler equations by finite volume methods using Runge-Kutta time stepping schemes. In: AIAA Paper 81-1259.

Ji, B., Luo, X., Peng, X., Wu, Y., Xu, H., 2012. Numerical anaysis of cavitation evolution and excited pressure fluctuation around a propeller in a non-uniform wake. Int. Journal of Multiphase Flow (43), 13-21.

Kunz, R., Boger, D., Stinebring, D., Chyczewski, T., Lindau, J., Gibeling, H., Venkateswaran, S., Govindan, T., 2000. A preconditioned Navier-Stokes method for two-phase flows with application to cavitation prediction. Computers \& Fluids $29(8), 849-875$.

Liu, T., Khoo, B., Xie, W., 2004. Isentropic one-fluid modelling of unsteady cavitating flow. Journal of Computational Physics 201 (1), 80-108.

Liu, T., Khoo, B., Yeo, K., 2001. The simulation of compressible multi-medium flow, II: applications to 2D underwater shock refraction. Computers and Fluids $30,219-314$. 
Merci, B., Steelant, J., Vierendeels, J., Riemslagh, K., Dick, E., 2000. Computational treatment of source terms in two-equation turbulence models. AIAA Journal 38 (11), 2085-2093.

Merkle, C., Feng, J., Buelow, P., 1998. Computation modeling of the dynamics of sheet cavitation. In: $3^{\text {rd }}$ International Symposium on Cavitation CAV1998, Grenoble, France.

Morgut, M., Nobile, E., Bilus, I., 2011. Comparison of mass transfer models for the numerical prediction of sheet cavitation around a hydrofoil. Int. Journal of Multiphase Flow 37, 620-626.

Muller, S., Helluy, P., Ballmann, J., 2010. Numerical simulation of a single bubble by compressible two-phase fluids. Int. Journal for Numerical Methods in Fluids $62(6), 591-631$.

Nourgaliev, R., Dinh, T., Theofanous, T., 2006. Adaptive characteristics-based matching for compressible multifluid dynamics. Journal of Computational Physics 213, 500-529.

Patella, R. F., Barre, S., Reboud, J., September 11-15 2006. Experiments and modelling of cavitating flows in Venturi : part II, unsteady cavitation. In: 6th International Symposium on Cavitation CAV2006, Wageningen, The Netherlands.

Petitpas, F., Massoni, J., Saurel, R., Lapedie, E., Munier, L., 2009. Diffuse interface model for high speed cavitating underwater systems. Int. Journal of Multiphase Flow 35, 747-759.

Ranjan, D., Oakley, J., Bonazza, R., 2011. Shock-bubble interactions. Annual review of Fluid Mechanics 43, 117-140. 
Reboud, J.-L., Stutz, B., Coutier, O., 1998. Two-phase flow structure of cavitation: experiment and modelling of unsteady effects. In: $3^{\text {rd }}$ International Symposium on Cavitation CAV1998, Grenoble, France.

Roe, P., 1981. Approximate Riemann solvers, parameters vectors, and difference schemes. Journal of Computational Physics 43, 357-372.

Saurel, R., Cocchi, J., Butler, P. B., 1999. Numerical study of cavitation in the wake of a hypervelocity underwater projectile. Journal of Propulsion and Power 15 (3), $513-522$.

Schmidt, D., Rutland, C., Corradini, M., 1999. A fully compressible, two-dimensional model of small, high-speed, cavitating nozzles. Atomization and Sprays 9, 255-276.

Schmidt, S., Sezal, I., Schnerr, G., September 2006. Compressible simulation of highspeed hydrodynamics with phase change. In: European Conference on Computational Fluid Dynamics ECCOMAS 2006, Delft, The Netherlands.

Senocak, I., Shyy, W., 2002. A pressure-based method for turbulent cavitating flow computations. Journal of Computational Physics 176 (2), 363-383.

Singhal, A., Athavale, M., Li, H., Jiang, Y., 2002. Mathematical basis and validation of the full cavitation model. Journal of Fluids Engineering 124 (3), 617-624.

Sinibaldi, E., Beux, F., Salvetti, M., 2006. A numerical method for 3D barotropic flows in turbomachinery. Flow Turbulence Combustion 76, 371-381.

Spalart, P., Allmaras, S., January 6-9 1992. A one-equation turbulence model for aerodynamic flows. In: AIAA 92-0439, 30 ${ }^{\text {th }}$ Aerospace Sciences Meeting - Reno, Nevada. 
Srinivasan, V., Salazar, A., Saito, K., 2009. Numerical simulation of cavitation dynamics using a cavitation-induced-momentum-defect (cimd) correction approach. Applied Mathematical Modelling 33 (3), 1529-1559.

Tatsumi, S., Martinelli, L., Jameson, A., 1995. Flux-limited schemes for the compressible Navier-Stokes equations. AIAA Journal 33 (2), 252-261.

Turkel, E., 1987. Preconditioned methods for solving the incompressible and low speed compressible equations. Journal of Computational Physics 172 (2), 277-298.

Venkateswaran, S., Lindau, J., Kunz, R., Merkle, C., 2002. Computation of multiphase mixture flows with compressibility effects. Journal of Computational Physics $180(1), 54-77$.

Ventikos, Y., Tzabiras, G., 2000. A numerical method for the simulation of steady and unsteady cavitating flows. Computers \& Fluids 29 (1), 63-88.

Viegas, J., Rubesin, M., July 12-14 1983. Wall-function boundary conditions in the solution of the Navier-Stokes equations for complex compressible flows. In: AIAA 83-1694, 16 ${ }^{\text {th }}$ Fluid and Plasma Dynamics Conference - Danver, Massachussetts.

Vortmann, C., Schnerr, G., Seelecke, S., 2003. Thermodynamic modeling and simulation of cavitating nozzle flow. Int. Journal of Heat and Fluid Flow 24, 774-783.

Wallis, G., 1967. One-dimensional two-phase flow. New York: McGraw-Hill.

Wang, G., Ostoja-Starzewski, M., 2007. Large eddy simulation of sheet/cloud cavitation on a NACA0015 hydrofoil. Applied Mathematical Modelling 31 (3), 417-447. 
Wu, J., Wang, G., Shyy, W., 2005. Time-dependent turbulent cavitating flow computations with interfacial transport and filter-based models. Int. Journal for $\mathrm{Nu}$ merical Methods in Fluids 49 (7), 739-761.

Xie, W., Liu, T., Khoo, B., 2007. The simulation of cavitating flows induced by underwater shock and free surface interaction. Applied Numerical Mathematics $57,734-745$.

Zein, A., Hantke, M., Warnecke, G., 2010. Modeling phase transition for compressible two-phase flows applied to metastable liquids. Journal of Computational Physics 229 (8), 2964-2998.

Zhou, L., Wang, Z., 2008. Numerical simulation of cavitation around a hydrofoil and evaluation of a RNG $k-\varepsilon$ model. Journal of Fluid Engineering 130 (1), 011302. 
Table 1: Parameters of the stiffened gas EOS for water at $T=355 \mathrm{~K}$.

\begin{tabular}{|c|c|c|c|c|c|}
\hline & $\gamma$ & $P_{\infty}(\mathrm{Pa})$ & $\mathrm{q}(\mathrm{J} / \mathrm{kg})$ & $C_{p}(\mathrm{~J} / \mathrm{K} . \mathrm{kg})$ & $\rho_{\text {sat }}\left(\mathrm{kg} / \mathrm{m}^{3}\right)$ \\
\hline liquid & 2.35 & $10^{9}$ & $-0.116710^{7}$ & 4267 & 1149.9 \\
\hline vapor & 1.43 & 0 & $0.203010^{7}$ & 1487 & 0.31 \\
\hline
\end{tabular}


Table 2: Geometrical data and flow configuration, Venturi geometries

\begin{tabular}{lcc}
\hline experimental parameters & case 1 & case 2 \\
\hline angle of the divergent & $4^{\circ}$ & $8^{\circ}$ \\
reference length $L_{r e f}$ & $252 \mathrm{~mm}$ & $224 \mathrm{~mm}$ \\
\hline inlet velocity $U_{\text {inlet }}$ & $10.8 \mathrm{~m} / \mathrm{s}$ & $7.04 \mathrm{~m} / \mathrm{s}$ \\
inlet pressure $P_{\text {inlet }}$ & $0.35 \mathrm{bar}$ & $0.52 \mathrm{bar}$ \\
cavitation parameter in the inlet section $\sigma_{\text {inlet }}$ & $0.547 \pm 0.05$ & $2.15 \pm 0.06$ \\
Reynolds number $R e_{L_{r e f}}$ & $2.710^{6}$ & $1.5710^{6}$ \\
\hline sheet cavity & smoothly fluctuating & self-oscillating \\
clouds shedding frequency & none & $45 \mathrm{~Hz}$ \\
time-averaged cavity length & $80 \mathrm{~mm}$ & - \\
\hline
\end{tabular}


Table 3: Parameters of the stiffened gas EOS for water at $T=293 \mathrm{~K}$.

\begin{tabular}{|c|c|c|c|c|c|}
\hline & $\gamma$ & $P_{\infty}(\mathrm{Pa})$ & $\mathrm{q}(\mathrm{J} / \mathrm{kg})$ & $C_{p}(\mathrm{~J} / \mathrm{K} . \mathrm{kg})$ & $\rho_{\text {sat }}\left(\mathrm{kg} / \mathrm{m}^{3}\right)$ \\
\hline liquid & 1.01 & $1.211 \quad 10^{7}$ & $-0.114210^{7}$ & 4183 & 998.16 \\
\hline vapor & 1.32 & 0 & $0.198510^{7}$ & 1883 & 0.0173 \\
\hline
\end{tabular}


Table 4: Numerical data and meshes

\begin{tabular}{lcc}
\hline numerical parameters & case 1 & case 2 \\
\hline meshes & $251 \times 62$ & $174 \times 56$ \\
\hline$y^{+}$values in first cells & 12 to 27 & 9 to 31 \\
\hline preconditioning parameter & 3 & 4 \\
CFL number & 0.4 & 0.3 \\
implicit Jacobi iterations & 15 & 15 \\
2nd and 4th order dissipation parameter & $1 ; 0.04$ & $1 ; 0.055$ \\
dual time stepping sub-iterations & 100 & 100 \\
dimensionless time step, $\Delta t^{*}=\frac{\Delta t U_{\text {inlet }}}{L_{\text {ref }}}$ & $1.9310^{-2}$ & $1.9110^{-3}$ \\
\hline
\end{tabular}


Table 5: Unsteady computations performed on the $8^{\circ}$ Venturi

\begin{tabular}{lll}
\hline$C_{\text {des }}$ & time-averaged $\sigma_{\text {inlet }}$ & frequency $(\mathrm{Hz})$ \\
\hline 0 & 2.18 & $44 \mathrm{~Hz}$ \\
1 & 2.19 & $42 \mathrm{~Hz}$ \\
4 & 2.23 & $40 \mathrm{~Hz}$ \\
6 & 2.22 & $36 \mathrm{~Hz}$ \\
8 & 2.25 & $25 \mathrm{~Hz}$ \\
10 & 2.24 & $16 \mathrm{~Hz}$ \\
20 & 2.15 & $7.5 \mathrm{~Hz}$ \\
\hline
\end{tabular}




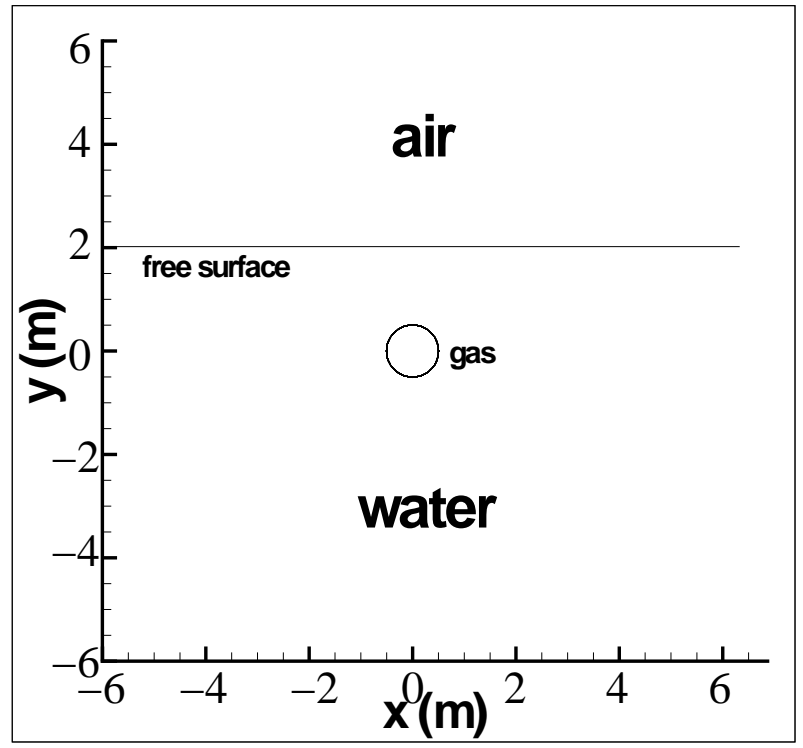

Figure 1: Underwater explosion, initial. 


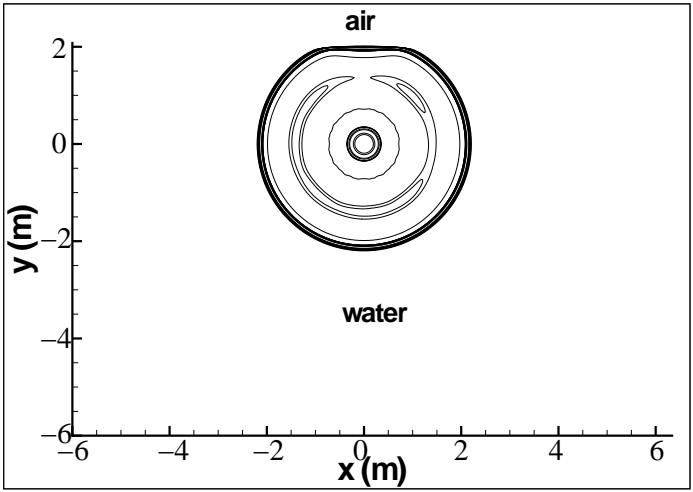

a)

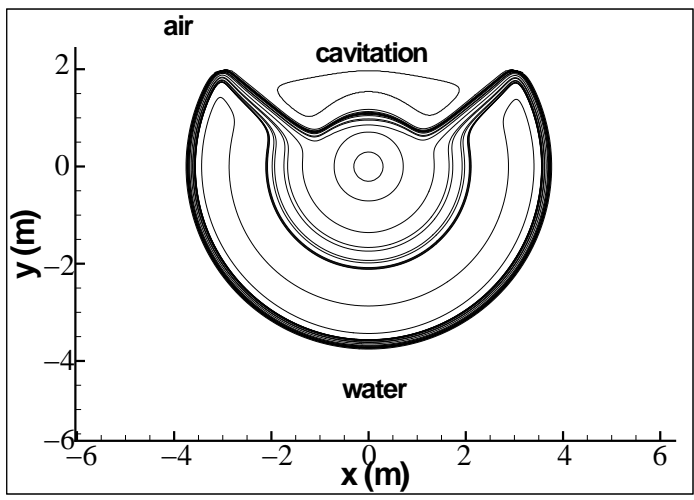

c)

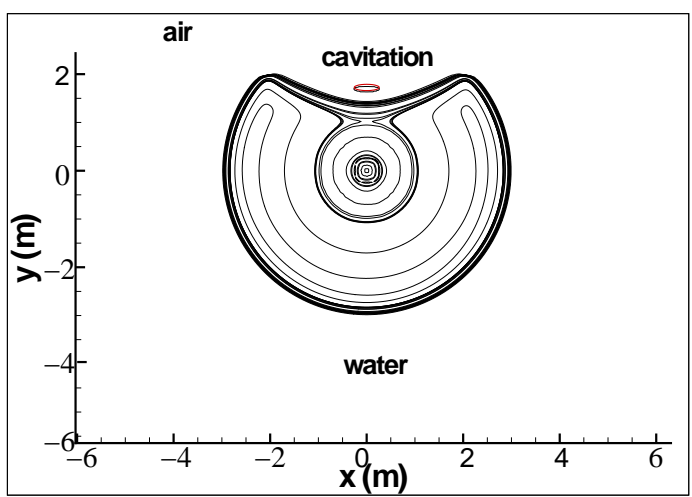

b)

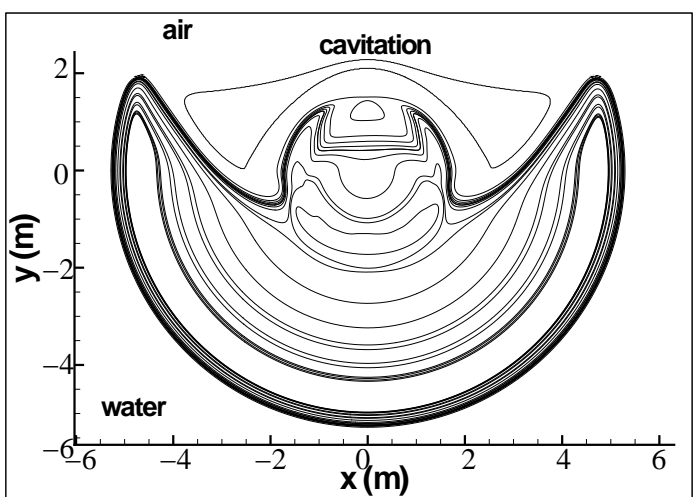

d)

Figure 2: Underwater explosion. 


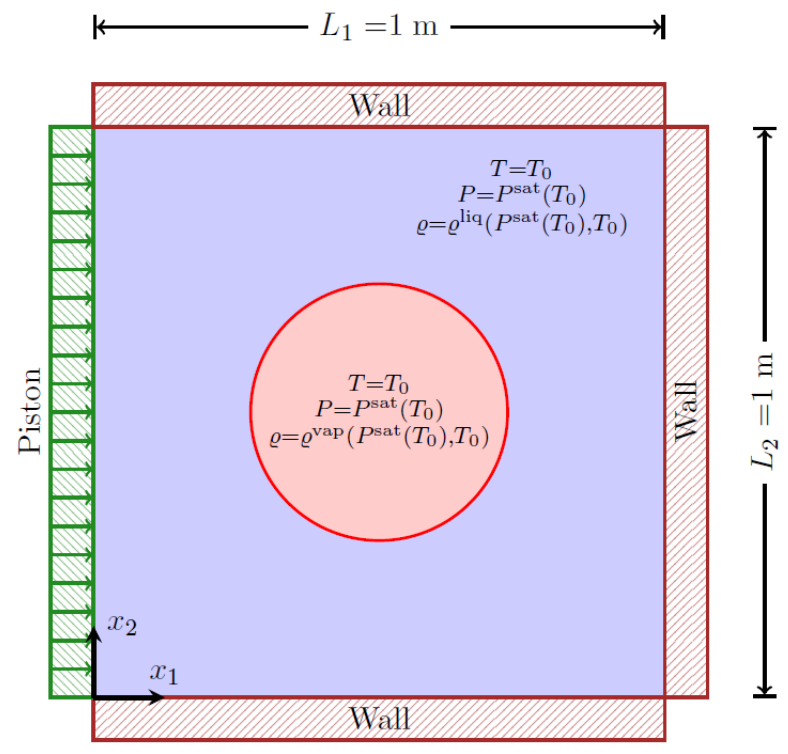

Figure 3: Compression of a vapour bubble, $\mathrm{t}=0$ (extracted from Faccanoni et al. (2012)). 

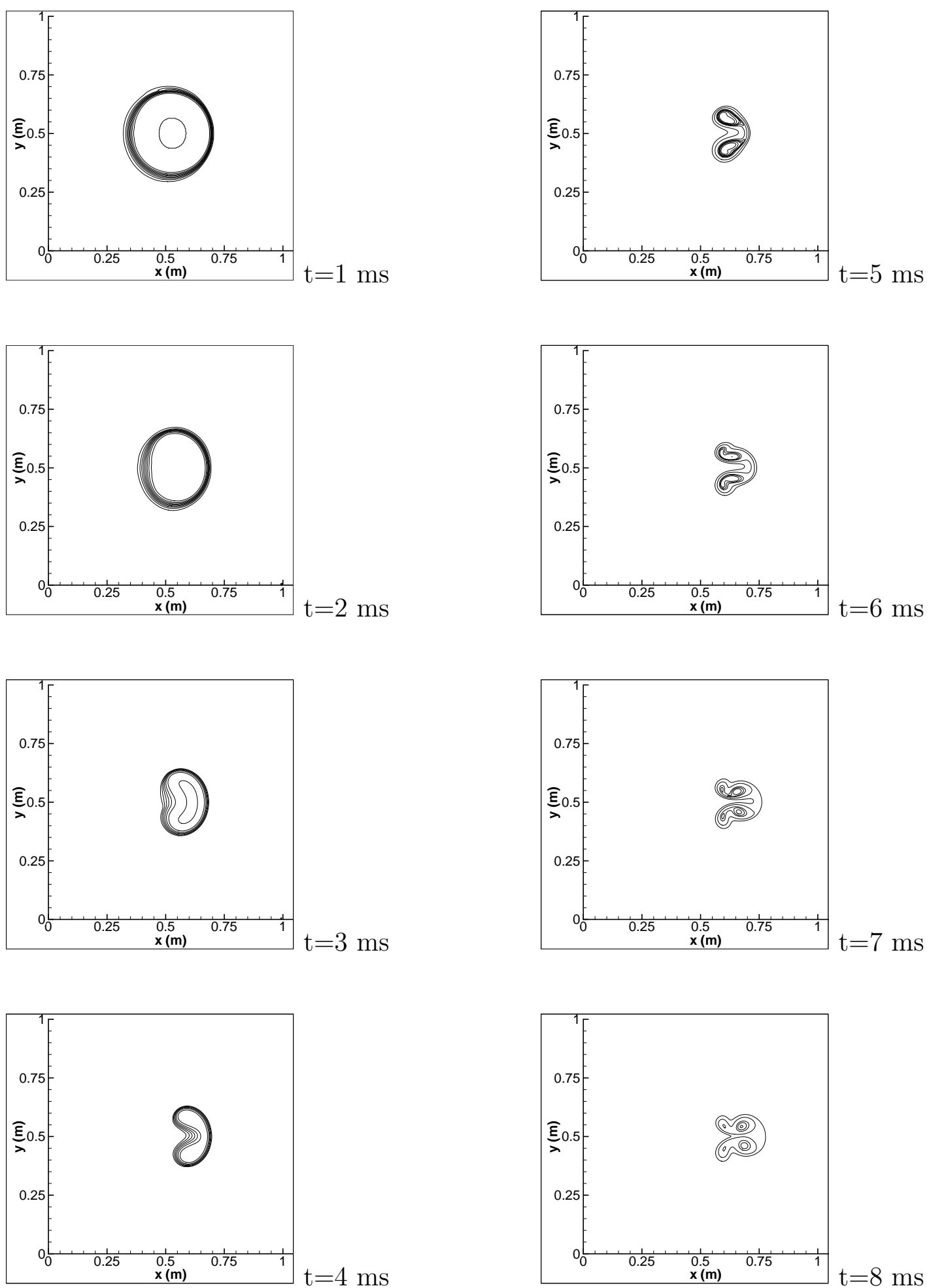

46

Figure 4: Time evolution of the volume fraction from $t=1 \mathrm{~ms}$ to $\mathrm{t}=8 \mathrm{~ms}$, compression of a vapour bubble. 

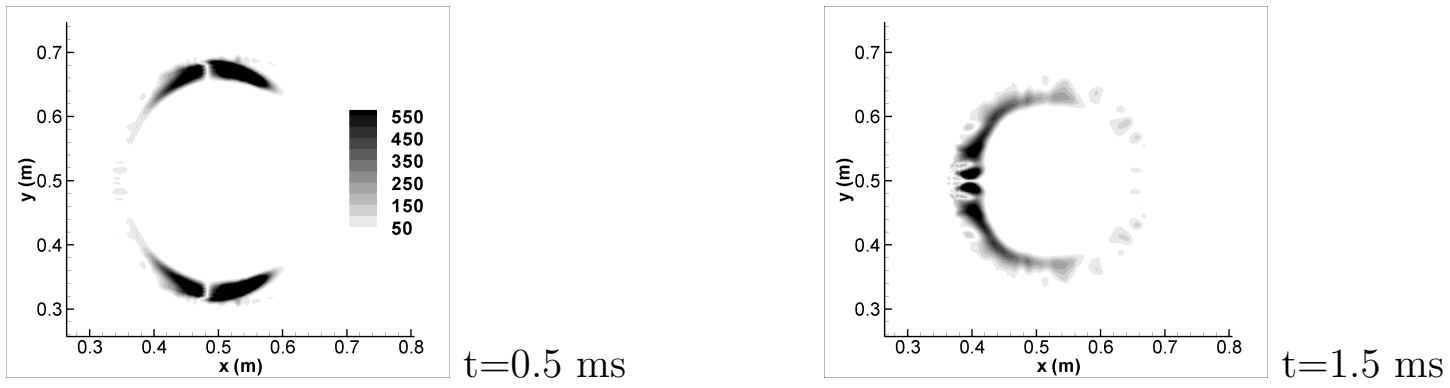

Figure 5: Modulus of the baroclinic torque, times $0.5 \mathrm{~ms}$ and $1.5 \mathrm{~ms}$, compression of a vapour bubble. 


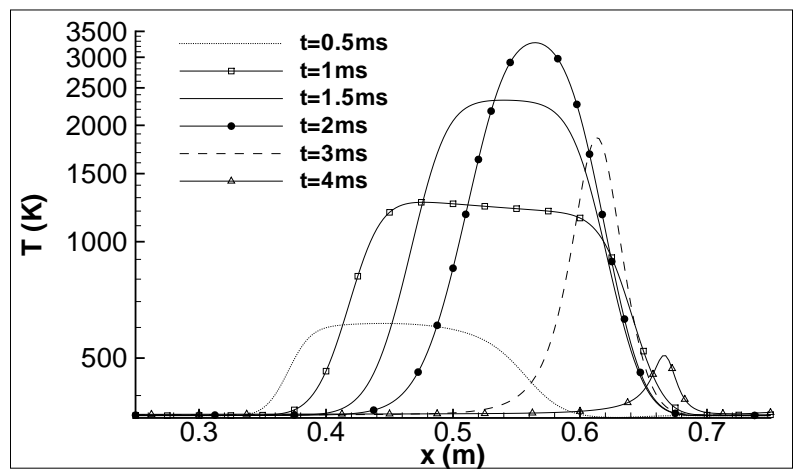

Figure 6: Evolution of the mixture temperature profile at position $y=0.5 \mathrm{~m}$, compression of a vapour bubble. 


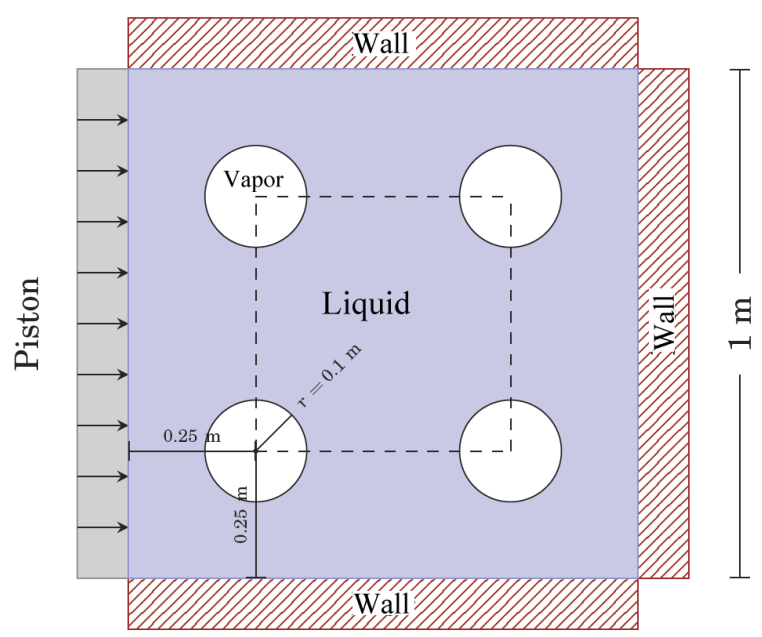

Figure 7: Compression of four vapour bubbles, $\mathrm{t}=0$ (extracted from Faccanoni et al. (2012)). 

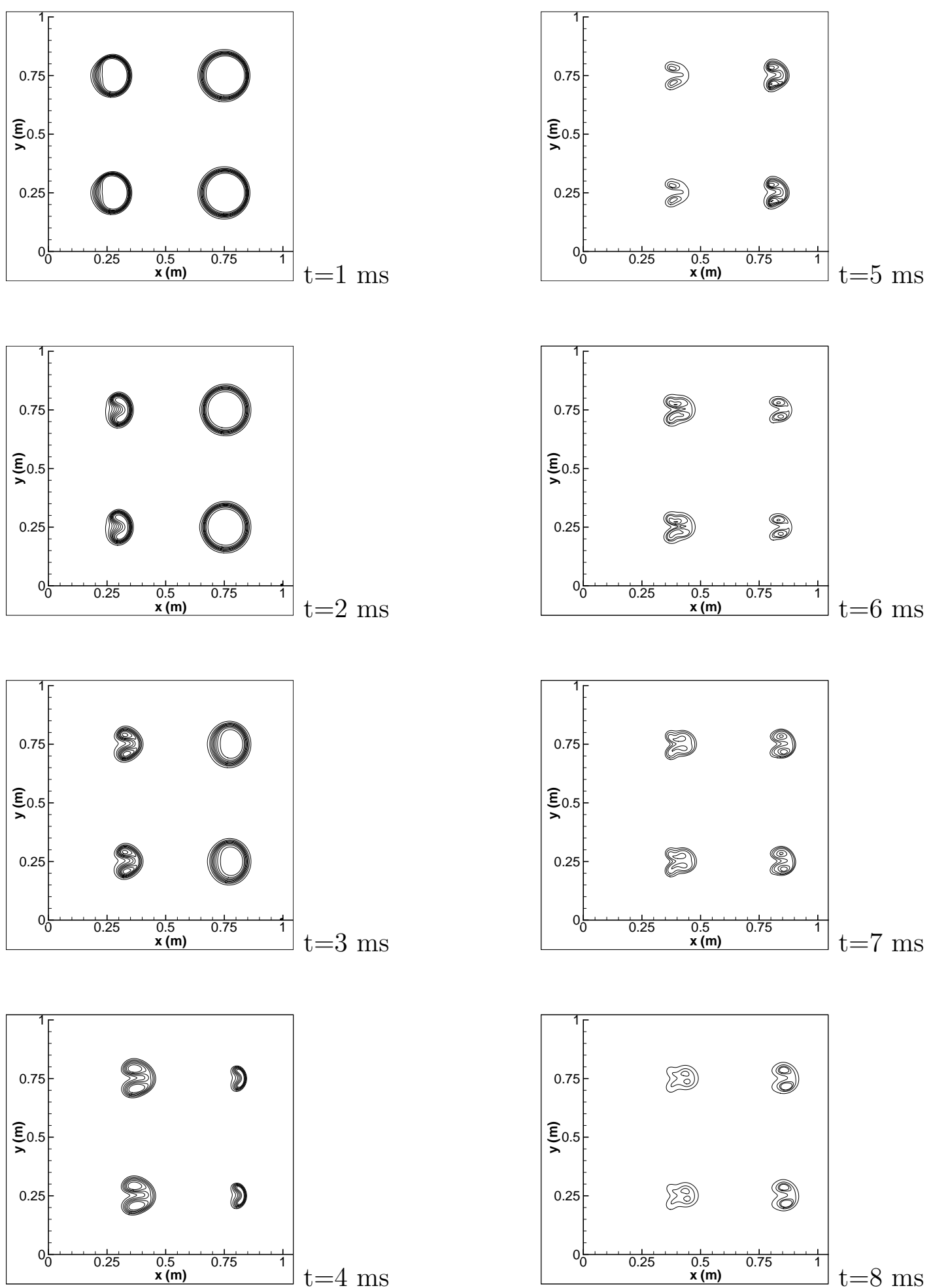

50

Figure 8: Time evolution of the volume fraction from $t=1 \mathrm{~ms}$ to $t=8 \mathrm{~ms}$, compression of four vapour bubbles. 

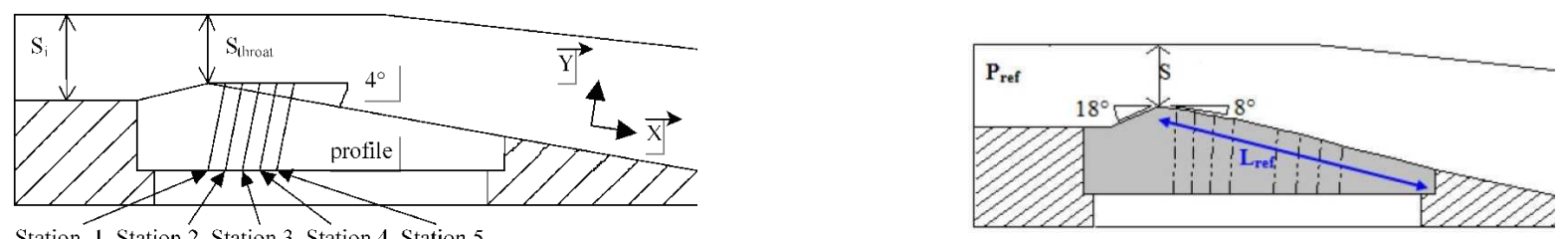

Figure 9: Venturi design and probe locations, $4^{\circ}$ divergence angle (left) and $8^{\circ}$ angle (right). 

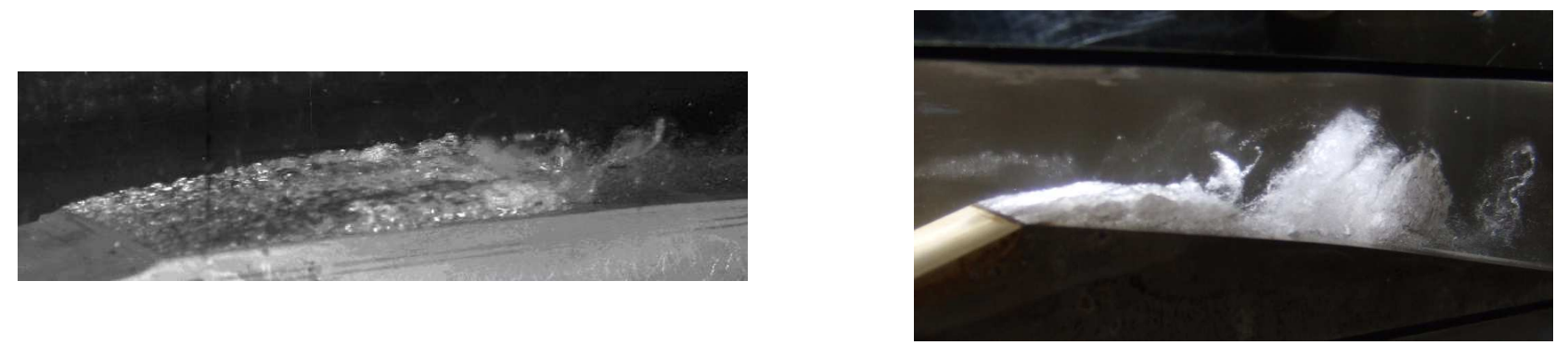

Figure 10: Photograph of cavities, $4^{\circ}$ Venturi (left) and $8^{\circ}$ Venturi (right). 


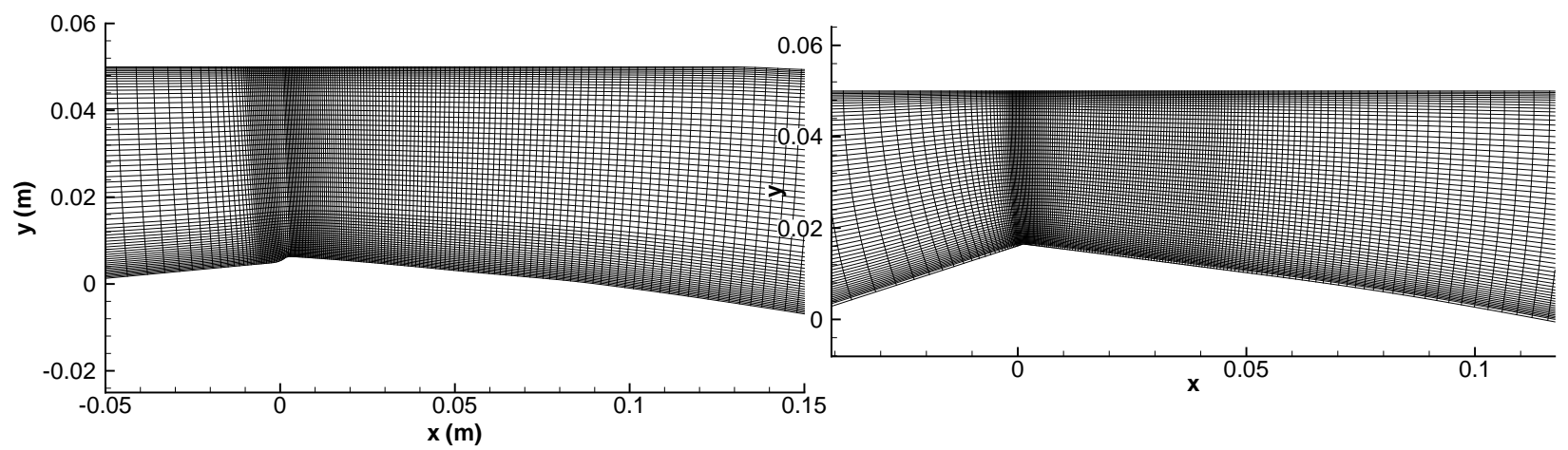

Figure 11: Enlargement of the mesh near the Venturi throat, $4^{\circ}$ Venturi (left) and $8^{\circ}$ Venturi (right). 

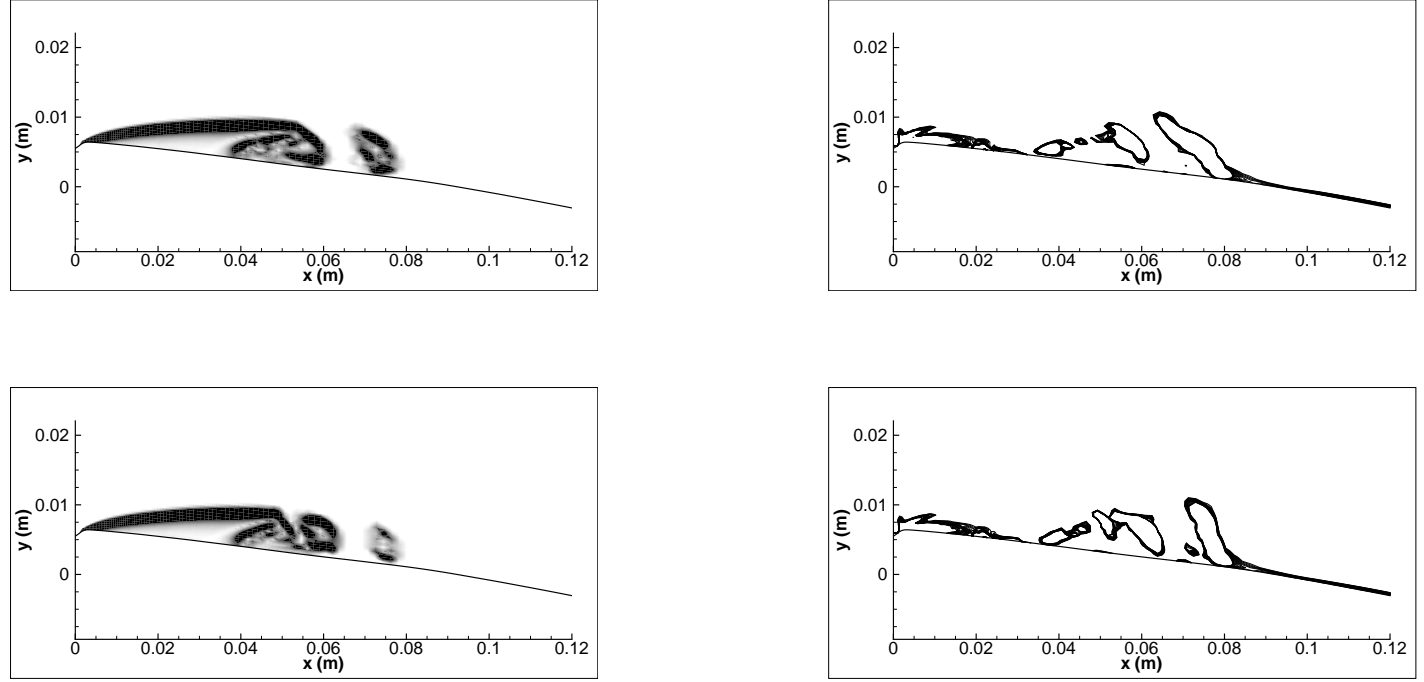

Figure 12: Dimensionless Q-criterion and density gradient modulus at two instants, $4^{\circ}$ Venturi. 

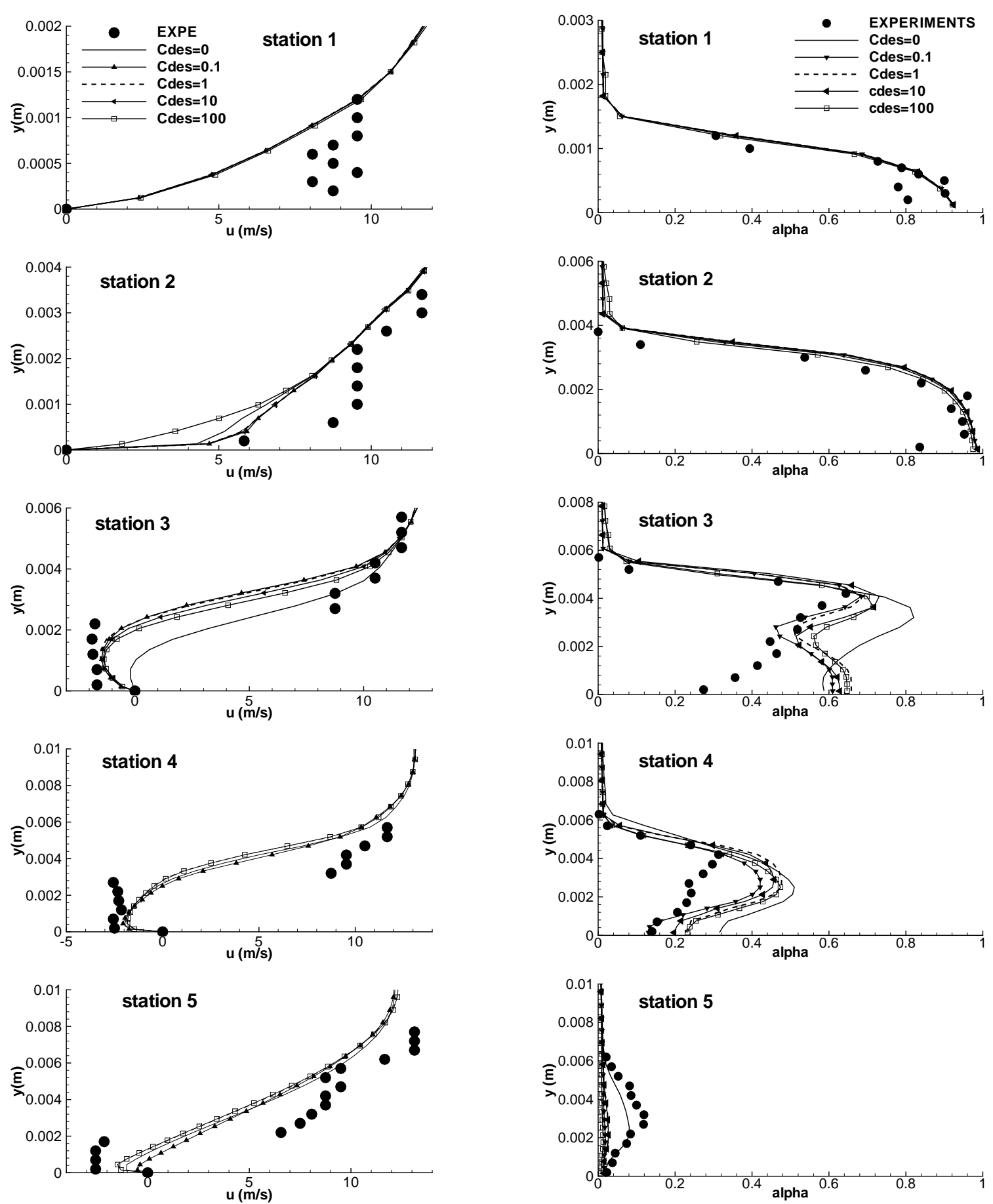

Figure 13: Time-averaged velocity (right) and void ratio (left) profiles from station 1 to $5,4^{\circ}$ Venturi. 


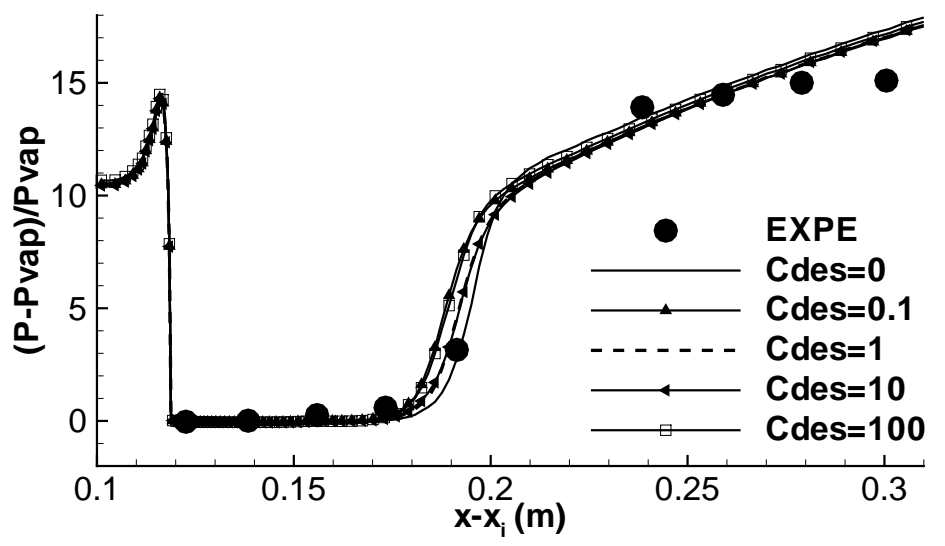

Figure 14: Time-averaged wall pressure evolution, $4^{\circ}$ Venturi. 


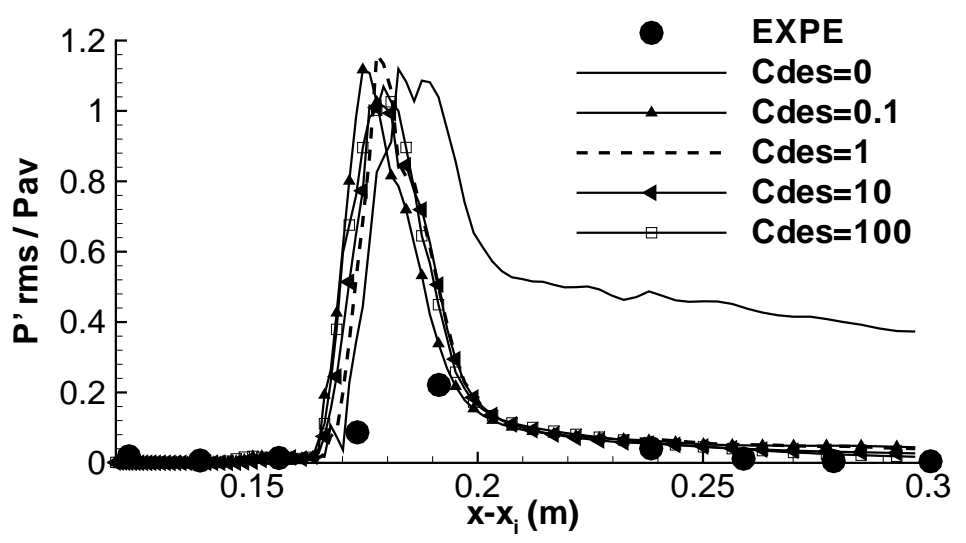

Figure 15: RMS wall pressure fluctuations, $4^{\circ}$ Venturi. 

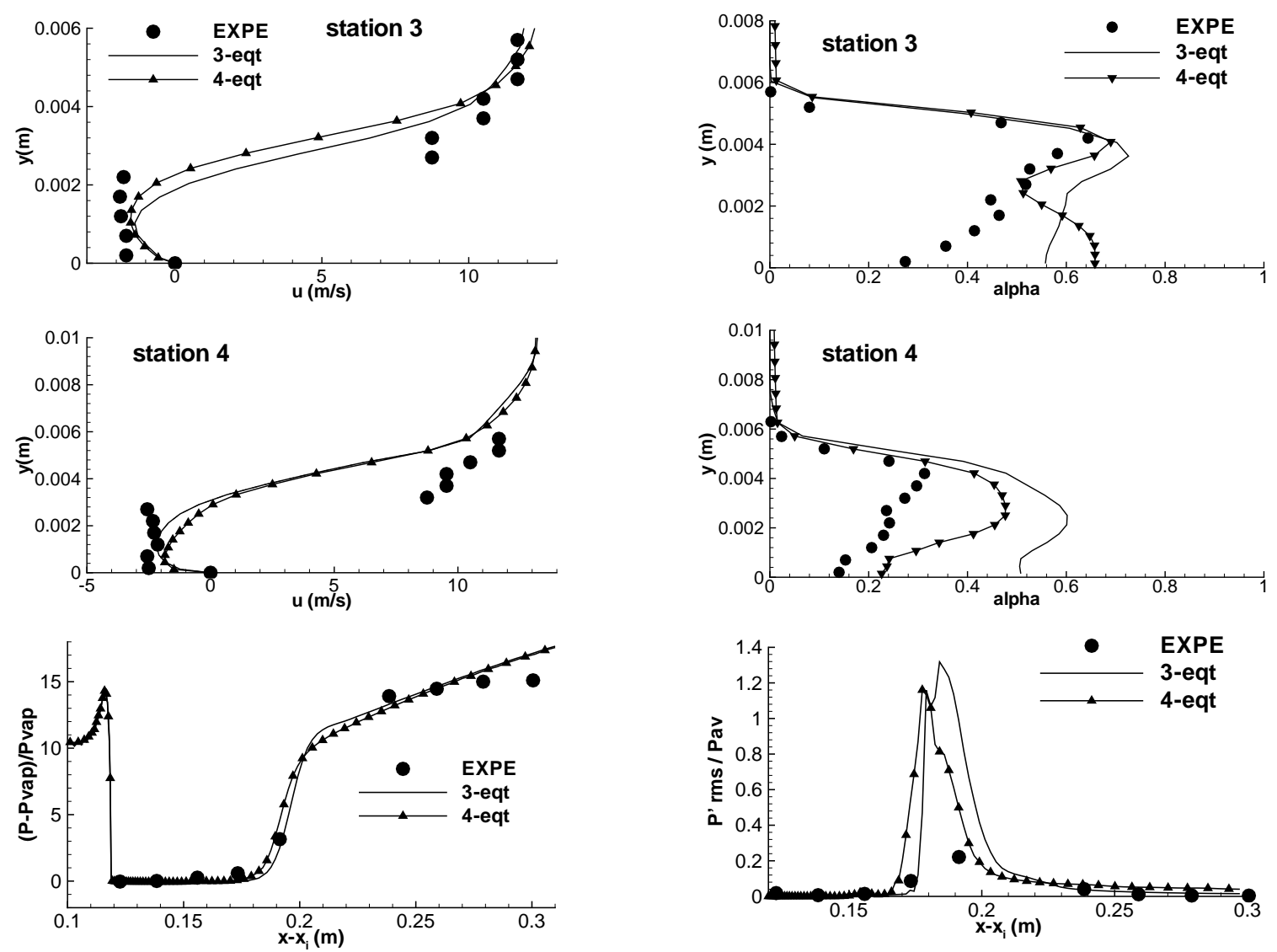

Figure 16: Models comparison: 3 -equation versus 4-equation with $C_{d e s}=1$, void ratio, velocity and pressure, $4^{\circ}$ Venturi. 

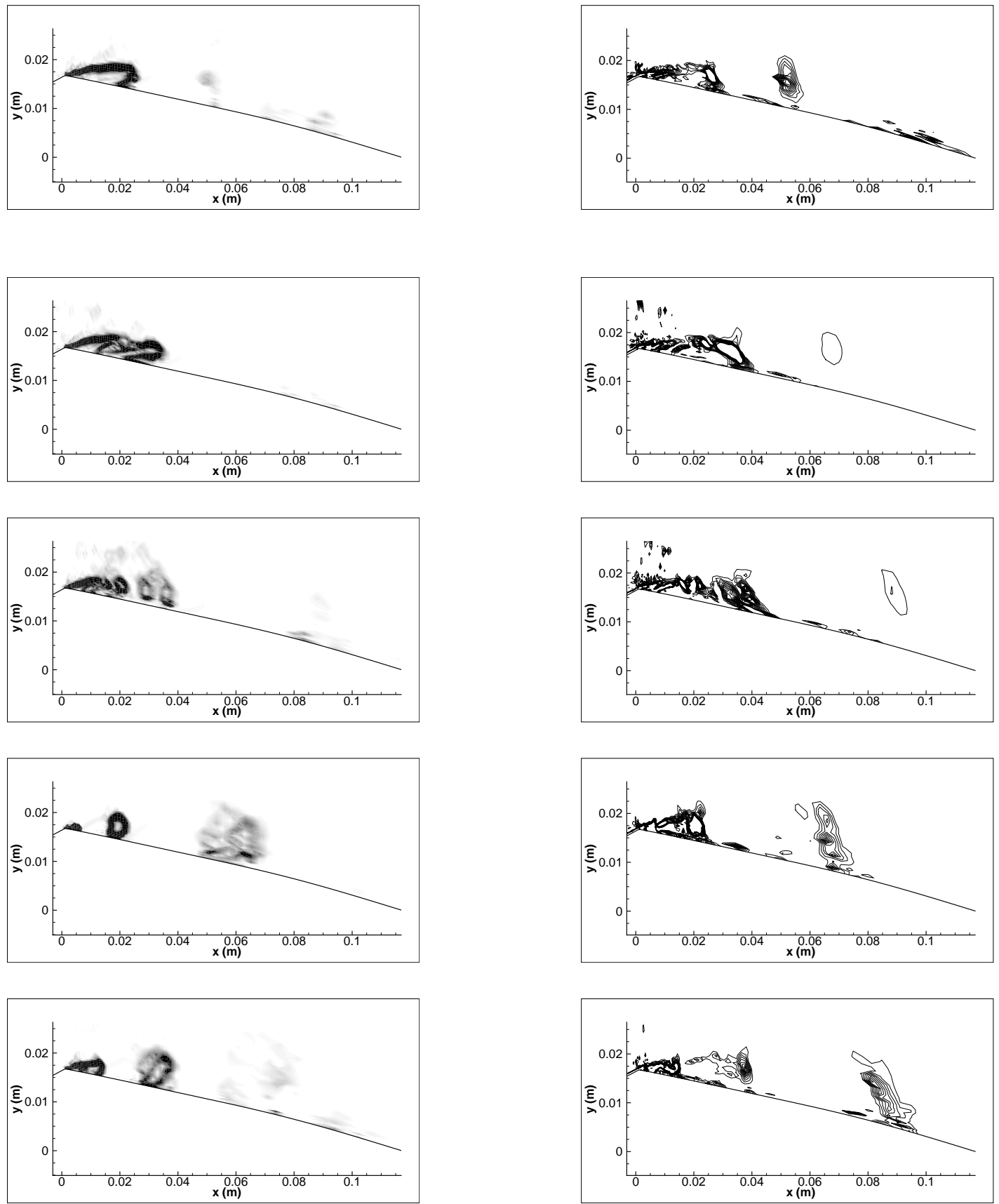

Figure 17: Density gradient modulus (left) and dimensionless Q-criterion (right) during one period, $C_{d e s}=1,8^{\circ}$ Venturi. 

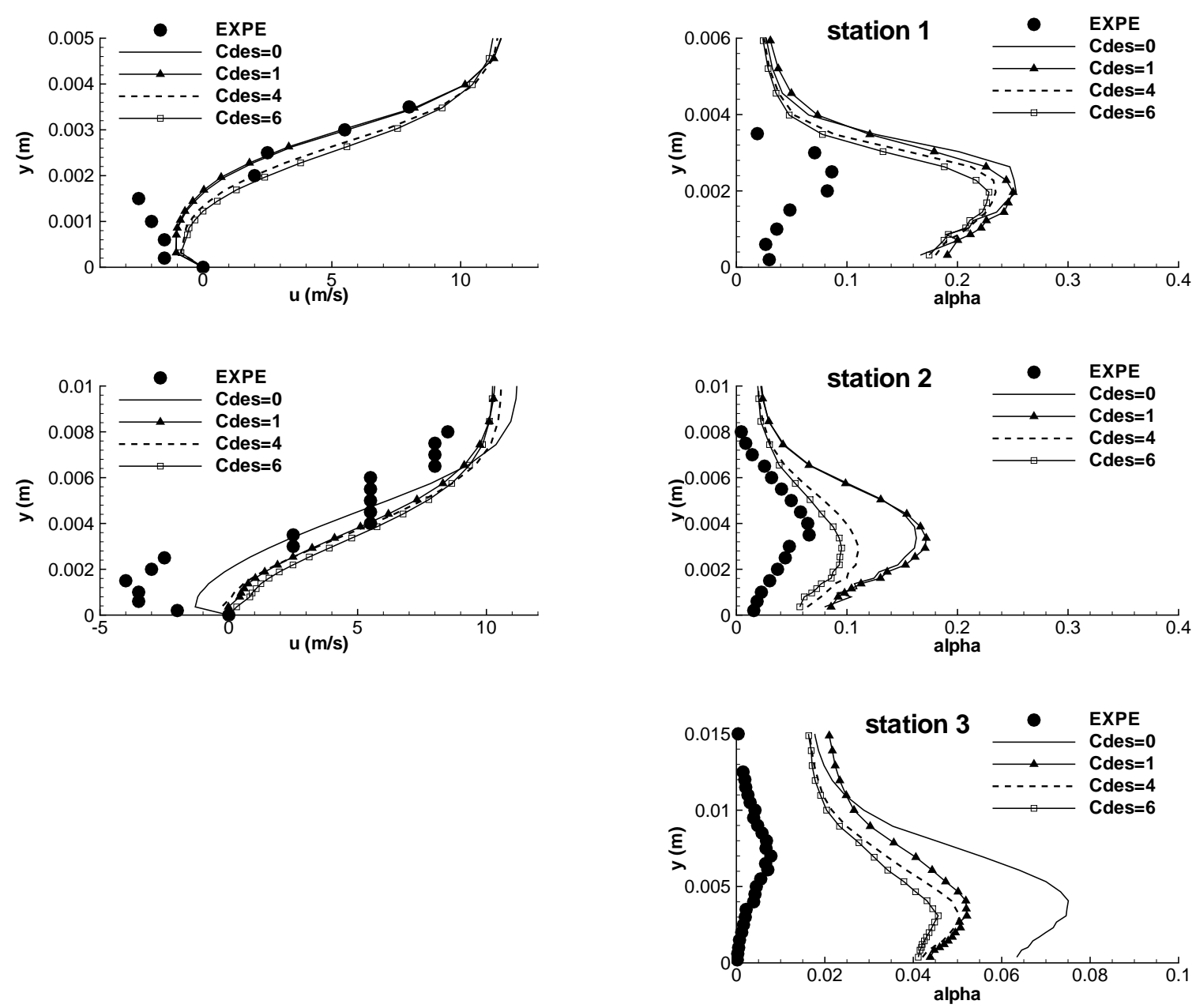

Figure 18: Time-averaged velocity (left) and void ratio (right) profiles from station 1 to $3,8^{\circ}$ Venturi. 


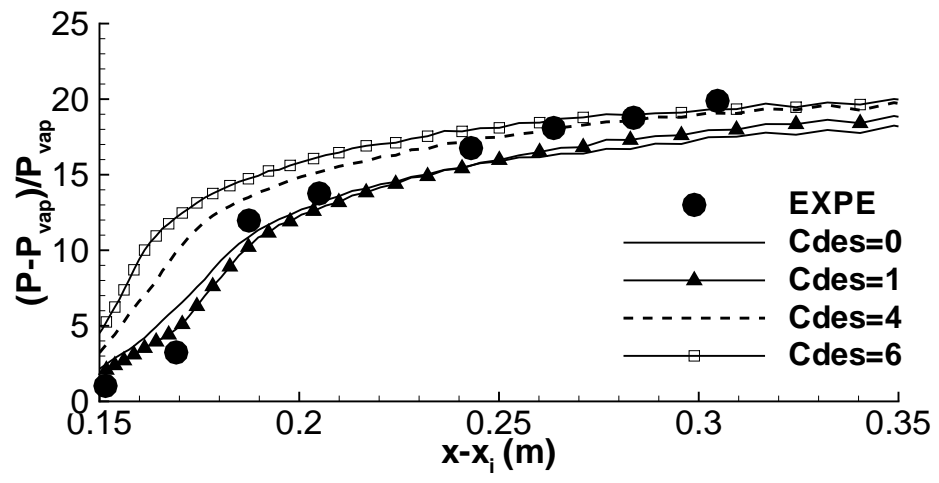

Figure 19: Time-averaged wall pressure evolution, $8^{\circ}$ Venturi. 

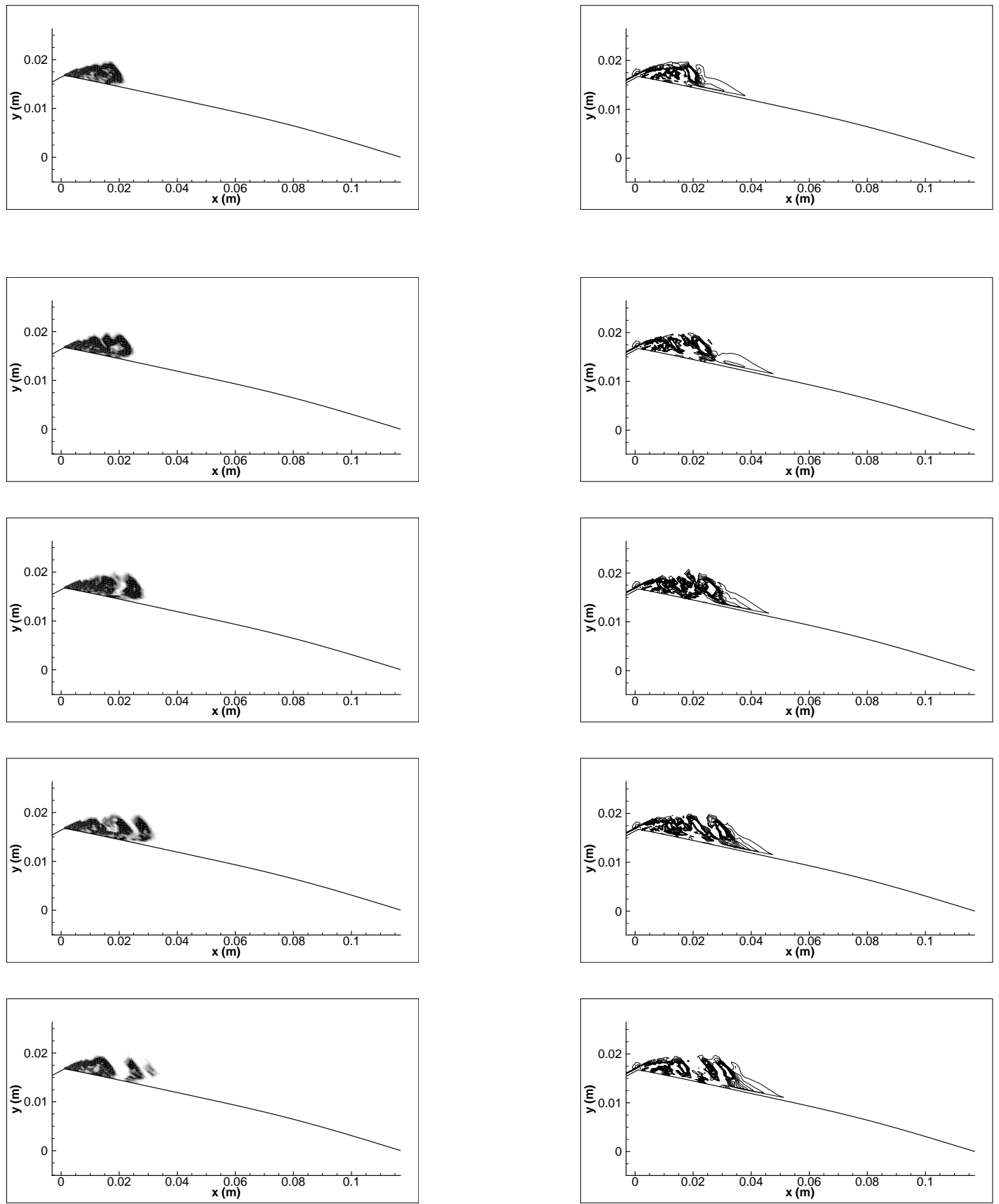

Figure 20: Density gradient modulus (left) and dimensionless Q-criterion (right) during one period, barotropic 3-equation model, $8^{\circ}$ Venturi. 

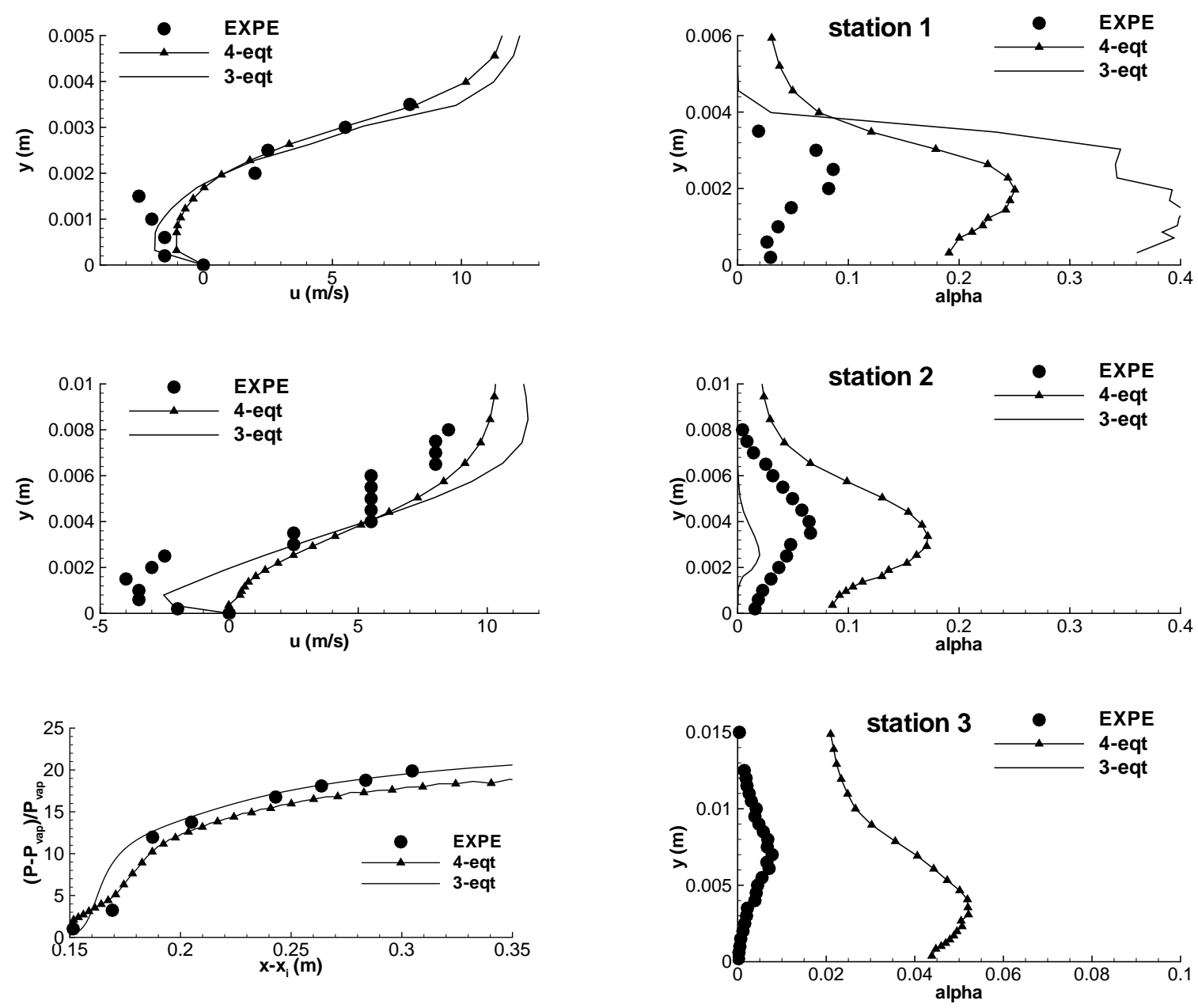

Figure 21: Models comparison: 3 -equation versus 4 -equation with $C_{d e s}=1$, void ratio, velocity and pressure, $8^{\circ}$ Venturi. 